\title{
A novel characterization of cubic Hamiltonian graphs via the associated quartic graphs*
}

\author{
Simona Bonvicini ${ }^{\dagger}$ \\ Department of Sciences and Methods for Engineering, University of Modena and Reggio \\ Emilia, Italy \\ Tomaž Pisanski \\ University of Primorska, Koper and University of Ljubljana, Ljubljana, Slovenia
}

Received 16 August 2015, accepted 18 December 2015, published online 6 March 2016

\begin{abstract}
We give a necessary and sufficient condition for a cubic graph to be Hamiltonian by analyzing Eulerian tours in certain spanning subgraphs of the quartic graph associated with the cubic graph by 1-factor contraction. This correspondence is most useful in the case when it induces a blue and red 2-factorization of the associated quartic graph. We use this condition to characterize the Hamiltonian $I$-graphs, a further generalization of generalized Petersen graphs. The characterization of Hamiltonian $I$-graphs follows from the fact that one can choose a 1 -factor in any $I$-graph in such a way that the corresponding associated quartic graph is a graph bundle having a cycle graph as base graph and a fiber and the fundamental factorization of graph bundles playing the role of blue and red factorization. The techniques that we develop allow us to represent Cayley multigraphs of degree 4, that are associated to abelian groups, as graph bundles. Moreover, we can find a family of connected cubic (multi)graphs that contains the family of connected $I$-graphs as a subfamily.

Keywords: Generalized Petersen graphs, I-graphs, Hamiltonian cycles, Eulerian tours, Cayley multigraphs.
\end{abstract}

Math. Subj. Class.: 05C45, 05C25, 05C15, 05C76, 05C70, 55R10, 05C60

\footnotetext{
* Research supported in part by the ARRS Grants P1-0294, N1-0032, and J1-6720.

${ }^{\dagger}$ Corresponding author

E-mail addresses: simona.bonvicini@unimore.it (Simona Bonvicini), Tomaz.Pisanski@fmf.uni-lj.si (Tomaž Pisanski)
} 


\section{Introduction}

A graph is Hamiltonian if it contains a spanning cycle (Hamiltonian cycle). To find a Hamiltonian cycle in a graph is an NP-complete problem (see [12]). This fact implies that a characterization result for Hamiltonian graphs is hard to find. For this reason, most graph theorists have restricted their attention to particular classes of graphs.

In this paper we consider cubic graphs. In Section 2 we give a necessary and sufficient condition for a cubic graph to be Hamiltonian. Using this condition we can completely characterize the Hamiltonian $I$-graphs.

The family of $I$-graphs is a generalization of the family of generalized Petersen graphs. In [5], the generalized Petersen graphs were further generalized to $I$-graphs. Let $n, p, q$ be positive integers, with $n \geq 3,1 \leq p, q \leq n-1$ and $p, q \neq n / 2$. An $I$-graph $I(n, p, q)$ has vertex-set $V(I(n, p, q))=\left\{v_{i}, u_{i}: 0 \leq i \leq n-1\right\}$ and edge-set $E(I(n, p, q))=$ $\left\{\left[v_{i}, v_{i+p}\right],\left[v_{i}, u_{i}\right],\left[u_{i}, u_{i+q}\right]: 0 \leq i \leq n-1\right\}$ (subscripts are read modulo $n$ ). The graph $I(n, p, q)$ is isomorphic to the graphs $I(n, q, p), I(n, n-p, q)$ and $I(n, p, n-q)$. It is connected if and only if $\operatorname{gcd}(n, p, q)=1$ (see [3]).

For $p=1$ the $I$-graph $I(n, 1, q)$ is known as a generalized Petersen graph and is denoted by $G(n, q)$. The Petersen graph is $G(5,2)$. It has been proved that $I(n, p, q)$ is isomorphic to a generalized Petersen graph if and only if $\operatorname{gcd}(n, p)=1$ or $\operatorname{gcd}(n, q)=1$ (see [3]). A connected $I$-graph which is not a generalized Petersen graph is called a proper $I$-graph. Recently, the class of $I$-graphs has been generalized to the class of GI-graphs (see [6]).

It is well known that the Petersen graph is not Hamiltonian. A characterization of Hamiltonian generalized Petersen graphs was obtained by Alspach [2].

Theorem 1.1 (Alspach, [2]). A generalized Petersen graph $G(n, q)$ is Hamiltonian if and only if it is not isomorphic to $G(n, 2)$ when $n \equiv 5(\bmod 6)$.

In this paper we develop a powerful theory that helps us to extend this result to all $I$-graphs.

Theorem 1.2. A connected I-graph is Hamiltonian if and only if it is not isomorphic to $G(n, 2)$ when $n \equiv 5(\bmod 6)$.

For the proof of the above main theorem, we developed techniques that are of interest by themselves and are presented in the following sections. In particular, we introduce good Eulerian graphs that are similar to lattice diagrams that were originally used by Alspach in his proof of Theorem 1.1.

Our theory also involves Cayley multigraphs. In Section 4 we show that Cayley multigraphs of degree 4, that are associated to abelian groups, can be represented as graph bundles [19]. By the results concerning the isomorphisms between Cayley multigraphs (see [7]), we can establish when two graph bundles are isomorphic or not (see Section 4.2). Combining the definition of graph bundles with Theorem 3.3, we can find a family of connected cubic (multi)graphs that contains the family of connected $I$-graphs as a subfamily (see Section 5). 


\section{Cubic graph with a 1-factor and the associated quartic graph with transitions}

A cubic Hamiltonian graph has a 1-factor. In fact, it has at least three (edge-disjoint) 1factors. Namely any Hamiltonian cycle is even and thus gives rise to two 1-factors and the remaining chords constitute the third 1-factor. The converse is not true. There are cubic graphs, like the Petersen graph, that have a 1-factor but are not Hamiltonian. Nevertheless, we may restrict our search for Hamiltonian graphs among the cubic graphs to the ones that possess a 1-factor. In this section, we give a necessary and sufficient condition for the existence of a Hamiltonian cycle in a cubic graph $G$ possessing a 1-factor $F$.

Let $G$ be a connected simple cubic graph and let $F$ be one of its 1 -factors. Denote by $X=G / F$ the graph obtained from $G$ by contracting the edges of $F$. The graph $X$ is connected, quartic, that is, regular of degree 4 and might have multiple edges ( $X$ has no loop since $G$ is simple). We say that the quartic graph $X$ is associated with $G$ and $F$. Since $X$ is even and connected, it is Eulerian. A path on three vertices with middle vertex $v$ that is a subgraph of $X$ is called a transition at $v$. Since any pair of edges incident at $v$ defines a transition, there are $\left(\begin{array}{l}4 \\ 2\end{array}\right)=6$ transitions at each vertex of $X$. For general graphs each vertex of valence $d$ gives rise to $\left(\begin{array}{l}d \\ 2\end{array}\right)$ transitions. In an Euler tour some transitions may be used, others are not used. We are interested in some particular Eulerian spanning subgraphs $W$. Note that any such graph is sub-quartic and the valence at any vertex of $W$ is either 4 or 2 . A vertex of valence 4 has therefore 6 transitions, while each vertex of valence 2 has $\left(\begin{array}{l}2 \\ 2\end{array}\right)=1$ transition. Let $Y$ be the complementary 2-factor of $F$ in $G$. Note that the edges of $Y$ are in one-to-one correspondence with the edges of $X$, while the edges of $F$ are in one-to-one correspondence with the vertices of $X$. If $a$ is an edge of $Y$, we denote by $a^{\prime}$ the corresponding edge in $X$. If $e$ is an edge of $F$, the corresponding vertex of $X$ will be denoted by $x_{e}$. Let $u$ and $v$ be the end-vertices of $e$ and let $a$ and $b$ be the other edges incident with $u$ and similarly $c$ and $d$ the edges incident with $v$. After contraction of $e$, the vertex $x_{e}$ is incident with four edges: $a^{\prime}, b^{\prime}, c^{\prime}, d^{\prime}$. By considering the pre-images of the six transitions at $x_{e}$, they fall into two disjoint classes. Transitions $a^{\prime} b^{\prime}$ and $c^{\prime} d^{\prime}$ are non-traversing while the other four transitions are traversing transitions. In the latter case the edge $e$ has to be used to traverse from one edge of the pre-image transition to the other.

Let $W$ be a spanning Eulerian subgraph of $X$. Transitions of $X$ carry over to $W$. The 4 -valent vertices of $W$ keep the same six transitions, while each 2 -valent vertex inherits a single transition. We say that $W$ is admissible if the transition at each 2 -valent vertex of $W$ is traversing.

Let $W$ be an admissible Eulerian subgraph of $X$. A closed walk in $W$ that allows only non-traversing transitions at each 4 -valent vertex of $W$ is said to be a closed walk with allowed transitions. A closed walk with allowed transitions passing through a 4-valent vertex $x_{e}$ of $W$ might use both transitions $a^{\prime} b^{\prime}, c^{\prime} d^{\prime}$ or only one of the two non-traversing transitions. If it passes through a 2 -valent vertex of $W$, then it uses traversing transitions. Hence, the underlying graph of a closed walk with allowed transitions might be a cycle. A partition of the edge-set of $W$ into closed walks with allowed transitions is said to be a tour with allowed transitions. Each closed walk in the tour is a component of the tour.

Lemma 2.1. Let $G$ be a connected cubic graph with 1-factor F. There is a one-to-one correspondence between 2-factors $T$ of $G$ and admissible Eulerian subgraphs $W$ of $X=$ $G / F$ in such a way that the number of cycles of $T$ is the same as the number of components of a tour with allowed transitions in $W$. 
Proof. Let $T$ be a 2 -factor of $G$ and let $e=u v$ be an edge of the 1-factor $F$. Let $W$ be the projection of $T$ to $X=G / F$. We will use the notation introduced above. Hence the edge $e$ and its end-vertices $u$ and $v$ project to the same vertex $x_{e}$ of $X$. There are two cases:

Case 1: $e$ belongs to $T$. In this case exactly one other edge, say $a$, incident with $u$ and another edge, say $c$, incident with $v$ belong to $T$. The other two edges $(b$ and $d)$ do not belong to $T$. This means that $x_{e}$ is a 2 -valent vertex with traversing transition.

Case 2: $e$ does not belong to $T$. In this case both edges $a$ and $b$ incident with $u$ belong to $T$ and both edges $c$ and $d$ incident with $v$ belong to $T$. In this case $x_{e}$ is a 4 -valent vertex with non-traversing transitions.

Clearly, $W$ is an admissible Eulerian subgraph. Each component of the tour determined by $W$ with transitions gives back a cycle of $T$. The correspondence between $T$ and $W$ is therefore established.

An Eulerian tour in $W$ with allowed transitions is said to be good. An admissible subgraph $W$ of $X$ possessing a good Eulerian tour is said to be a good Eulerian subgraph. In a good Eulerian subgraph $W$ there are two extreme cases:

1. each vertex of $W$ is 4-valent: this means that $W=X$; in this case the complementary 2-factor $Y=G-F$ is a Hamiltonian cycle and no edge of $F$ is used;

2. each vertex of $W$ is 2-valent: this means that $W$ is a good Hamiltonian cycle in $X$. In this case $F$ together with the pre-images of edges of $W$ in $G$ form a Hamiltonian cycle.

Theorem 2.2. Let $G$ be a connected cubic graph with 1-factor F. Then $G$ is Hamiltonian if and only if $X=G / F$ contains a good Eulerian subgraph $W$.

Proof. Clearly $G$ is Hamiltonian if and only if it contains a 2-factor with a single cycle. By Lemma 2.1, this is true if and only if $W$ is an admissible Eulerian subgraph possessing an Eulerian tour with allowed transitions. But this means $W$ is good.

Corollary 2.3. Let $G$ be a connected cubic graph with 1-factor F. Finding a good Eulerian subgraph $W$ of $X=G / F$ is NP-complete.

Proof. Since finding a good Eulerian subgraph is equivalent to finding a Hamiltonian cycle in a cubic graph, and the latter is NP-complete [12], the result follows readily.

Also in [11] Eulerian graphs are used to find a Hamiltonian cycle (and other graph properties), but our method is different.

The results of this section may be applied to connected $I$-graphs. The obvious 1 factor $F$ of an $I$-graph $I(n, p, q)$ consists of spokes. Let $Q(n, p, q)$ denote the quotient $I(n, p, q) / F$. We will call $Q(n, p, q)$ the quartic graph associated with $I(n, p, q)$.

Corollary 2.4. Let $I(n, p, q)$ be a connected I-graph and let $Q(n, p, q)$ be its associated quartic graph. Then $I(n, p, q)$ is Hamiltonian if and only if $Q(n, p, q)$ contains a good Eulerian subgraph $W$. 


\section{Special 1-factors and their applications}

Let $G$ be a cubic graph, $F$ a 1-factor and $Y$ the complementary 2-factor of $F$ in $G$. Define an auxiliary graph $Y(G, F)$ having cycles of $Y$ as vertices and having two vertices adjacent if and only if the corresponding cycles of $Y$ are joined by one or more edges of $F$. If an edge of $F$ is a chord in one of the cycles of $Y$, then the graph $Y(G, F)$ has a loop. We shall say that the 1 -factor $F$ is special if the graph $Y(G, F)$ is bipartite. A cubic graph with a special 1-factor will be called special. If $F$ is a special 1-factor of $G$, then the edges of $F$ join vertices belonging to distinct cycles of $Y$ since $Y(G, F)$ is loopless.

Theorem 3.1. Let $G$ be a connected cubic graph with a 1-factor, and let $F$ be one of its 1 -factors and $X=G / F$ its associated quartic graph. Then $X$ admits a 2-factor whose edges may be colored blue or red in such a way that the traversing transitions are exactly color-switching and non-traversing transitions are color-preserving if and only if $G$ and $F$ are special.

Proof. Assume that $F$ is a special 1-factor of $G$. Since $Y(G, F)$ is bipartite, we can bicolor the vertices of $Y(G, F)$ : let one set of the bipartition be blue and the other red. This coloring induces a coloring on the edges of $Y$ : for every blue vertex (respectively, red vertex) of $Y(G, F)$ we color in blue (respectively, in red) the edges of the corresponding cycle of $Y$. Since the edges of $Y$ are in one-to-one correspondence with the edges of $X$, we obtain a 2 -factorization of $X$ into a blue 2 -factor and red 2-factor. Since $F$ is special, the edges of $F$ are incident with vertices of $G$ belonging to cycles of $Y$ with different colors (a blue cycle and a red cycle). Therefore, a traversing transition is color-switching and a non-traversing transition is color-preserving.

Conversely, assume that $X$ has a blue and red 2-factorization such that the traversing transitions are color-switching and non-traversing transitions are color-preserving. Since the edges of $X$ are in one-to-one correspondence with the edges of $Y$, we can partition the cycles of $Y$ into red cycles and blue cycles. Since the traversing transitions are colorswitching and non-traversing transitions are color-preserving, the edges of $F$ are incident with edges belonging to cycles of different colors. This means that the graph $Y(G, F)$ is bipartite, hence $F$ is special.

Proposition 3.2. Let $G$ and $F$ be special and let $W$ be any Eulerian subgraph of $X=G / F$ the associated quartic graph with a blue and red 2-factorization. Then $W$ is admissible if and only if each 2-valent vertex is incident with edges of different colors.

Proof. An Eulerian subgraph $W$ is admissible if and only if each 2-valent vertex $v$ in $W$ is incident with edges forming a traversing transition at $v$. By Theorem 3.1, a traversing transition is color-switching. Hence, $W$ is admissible if and only if the edges incident with $v$ have different colors.

Note that quartic graphs with a given 2-factorization can be put into one-to-one correspondence with special cubic graphs.

Theorem 3.3. Any special cubic graph $G$ with a special 1-factor $F$ gives rise to the associated quartic graph with a blue and red 2-factorization. However, any quartic graph with a given 2-factorization determines back a unique special cubic graph by color-preserving splitting vertices and inserting a special 1-factor. 
Proof. By Theorem 3.1, a special cubic graph $G$ with a special 1-factor $F$ gives rise to the graph $X=G / F$ admitting a blue and red 2-factorization.

Conversely, it is well known that every quartic graph $X$ possesses a 2 -factorization, that is, the edges of $X$ can be partitioned into a blue and red 2-factor. We use the blue and red 2-factors of $X$ to construct a cubic graph $G$ as follows: put in $G$ a copy of the blue 2-factor and a copy of the red 2-factor; construct a 1 -factor of $G$ by joining vertices belonging to distinct copies. It is straightforward to see that $G$ and $F$ are special.

We will now apply this theory to the $I$-graphs. In Section 7 we will see that this theory allows us to find a Hamiltonian cycle in a proper $I$-graph and also to find a family of special cubic graphs that contains the family of $I$-graphs as a subfamily (see Section 5 ).

Let $I(n, p, q)$ be an $I$-graph. A vertex $v_{i}$ (respectively, $u_{i}$ ) is called an outer vertex (respectively, an inner vertex). An edge of type $\left[v_{i}, v_{i+p}\right]$ (respectively, of type $\left[u_{i}, u_{i+q}\right]$ ) is called an outer edge (respectively, an inner edge). An edge $\left[v_{i}, u_{i}\right]$ is called a spoke. The spokes of $I(n, p, q)$ determine a 1 -factor of $I(n, p, q)$. The set of outer edges is called the outer rim, the set of inner edges is called the inner rim. As a consequence of the results proved in [3], the following holds.

Proposition 3.4. Let $I(n, p, q), n \geq 3,1 \leq p, q \leq n-1, p, q \neq n / 2$, be an I-graph. Set $t=\operatorname{gcd}(n, q)$ and $s=n / t$. Then $t<n / 2$ and $3 \leq s \leq n$. Moreover, $I(n, p, q)$ is connected if an only if $\operatorname{gcd}(t, p)=1$ and $\operatorname{gcd}(s, p)$ is coprime with $q$. It is proper if and only if $t$ and $\operatorname{gcd}(s, p)$ are different from 1 .

Proof. The integer $t$ satisfies the inequality $t<n / 2$, since $t$ is a divisor of $q$ and $q \leq n-1$, $q \neq n / 2$; whence $3 \leq s \leq n$. By the results proved in [3], $I(n, p, q)$ is connected if and only if $\operatorname{gcd}(n, p, q)=1$. Since $n=s t$ and $q=t(q / t)$, the relation $\operatorname{gcd}(n, p, q)=1$ can be written as $\operatorname{gcd}(s t, p, t(q / t))=1$, whence $\operatorname{gcd}(t, p)=1$ and $\operatorname{gcd}(s, p)$ is coprime with $q$. Also the converse is true, and therefore $I(n, p, q)$ is connected if and only if $\operatorname{gcd}(t, p)=1$ and $\operatorname{gcd}(s, p)$ is coprime with $q$. A connected $I$-graph $I(n, p, q)$ is a generalized Petersen graph if and only if $\operatorname{gcd}(n, q)=1$ or $\operatorname{gcd}(n, p)=1$ (see [3]). By the previous results, $I(n, p, q)$ is a generalized Petersen graph if and only if $1=\operatorname{gcd}(n, q)=t$ or $1=\operatorname{gcd}(n, p)=\operatorname{gcd}(s t, p)=\operatorname{gcd}(s, p)$. The assertion follows.

The smallest proper $I$-graphs are $I(12,2,3)$ and $I(12,4,3)$. It is straightforward to see that the following result holds.

Lemma 3.5. Let $F$ be the 1-factor determined by the spokes of $I(n, p, q)$ and $X=$ $Q(n, p, q)$ its associated quartic graph. Then $F$ is special, the graph $X$ is a circulant multigraph $\operatorname{Cir}(n ; p, q)$, the blue edges of $X$ correspond to the inner rim and the red edges to the outer rim of $I(n, p, q)$.

In the next section we introduce a class of graphs $X(s, t, r)$ and later show that it contains $Q(n, p, q)$ as its subclass.

\section{Graphs $X(s, t, r)$}

Let $\Gamma$ be a group in additive notation with identity element $0_{\Gamma}$. Let $S$ be a list of not necessarily distinct elements of $\Gamma$ satisfying the symmetry property $S=-S=\{-\gamma: \gamma \in S\}$. The Cayley multigraph associated with $\Gamma$ and $S$, denoted by $\operatorname{Cay}(\Gamma, S)$, is an undirected multigraph having the elements of $\Gamma$ as vertices and edges of the form $[x, x+\gamma]$ with 
$x \in \Gamma, \gamma \in S$. If $\Gamma$ is a cyclic group of order $n$, then $\operatorname{Cay}(\Gamma, S)$ is a circulant multigraph of order $n$. A Cayley multigraph $\operatorname{Cay}(\Gamma, S)$ is regular of degree $|S|$ (in determining $|S|$, each element of $S$ is considered according to its multiplicity in $S$ ). It is connected if and only if $S$ is a set of generators of $\Gamma$. If the elements of $S$ are pairwise distinct, then $\operatorname{Cay}(\Gamma, S)$ is a simple graph and we will use the term Cayley graph. We are interested in connected Cayley multigraphs of degree 4 . In this case we write $S$ as the list $S=\left\{ \pm \gamma_{1}, \pm \gamma_{2}\right\}$. A circulant multigraph of order $n$ will be denoted by $\operatorname{Cir}\left(n ; \pm \gamma_{1}, \pm \gamma_{2}\right)$. If $\gamma_{i}$, with $i \in\{1,2\}$, is an involution of $\Gamma$ or the trivial element, then $\pm \gamma_{i}$ means that the element $\gamma_{i}$ appears twice in the list $S$. Consequently, the associated Cayley multigraph has multiple edges or loops. We will denote by $o\left(\gamma_{i}\right)$ the order of $\gamma_{i}$. We will show that the Cayley multigraphs $\operatorname{Cay}\left(\Gamma,\left\{ \pm \gamma_{1}, \pm \gamma_{2}\right\}\right)$ defined on a suitable abelian group $\Gamma$ (and in particular the circulant multigraphs $\left.\operatorname{Cir}\left(n ; \pm \gamma_{1}, \pm \gamma_{2}\right)\right)$ can be given a different interpretation in terms of $X(s, t, r)$ graphs (see Figure 1) defined as follows.

Definition 1. Let $s, t \geq 1,0 \leq r \leq s-1$ be integers. Let $X(s, t, r)$ be the graph with vertex-set $\left\{x_{j}^{i}: 0 \leq i \leq t-1,0 \leq j \leq s-1\right\}$ and edge-set $\left\{\left[x_{j}^{i}, x_{j+1}^{i}\right]: 0 \leq i \leq t-1,0 \leq\right.$ $j \leq s-1\} \cup\left\{\left[x_{j}^{i}, x_{j}^{i+1}\right]: 0 \leq i \leq t-2,0 \leq j \leq s-1\right\} \cup\left\{\left[x_{j}^{t-1}, x_{j+r}^{0}\right]: 0 \leq j \leq s-1\right\}$ (the superscripts are read modulo $t$, the subscripts are read modulo $s$ ).

The graph $X(s, t, r)$ has edges of type $\left[x_{j}^{i}, x_{j+1}^{i}\right],\left[x_{j}^{i}, x_{j}^{i+1}\right]$ or $\left[x_{j}^{t-1}, x_{j+r}^{0}\right]$. An edge of type $\left[x_{j}^{i}, x_{j+1}^{i}\right]$ will be called horizontal. An edge of type $\left[x_{j}^{i}, x_{j}^{i+1}\right]$ will be called vertical, an edge of type $\left[x_{j}^{t-1}, x_{j+r}^{0}\right]$ will be called diagonal. For $t=1$, we say that the edges are horizontal and diagonal (a diagonal edge is an edge of type $\left[x_{j}^{0}, x_{j+r}^{0}\right]$ ). For $s=1$, the horizontal edges are loops. For $(t, r)=(1,0)$, the diagonal edges are loops. For $s=2$ or $s>2$ and $(t, r)=(1,1),(1, s / 2),(2,0)$ the graph has multiple edges. For the other values of $s, t, r$, the graph $X(s, t, r)$ is a simple graph. A simple graph $X(s, t, r)$ is a graph bundle with a cycle fiber $C_{s}$ over a cycle base $C_{t}$; the parameter $r$ represents an automorphism of the cycle $C_{s}$ that shifts the cycle $r$ steps (see [19] for more details on graph bundles). In the literature a simple graph $X(s, t, r)$ is also called $r$-pseudo-cartesian product of two cycles (see for instance [10]). The definition of $X(s, t, r)$ suggests that the graph $X(s, t, r)$ is isomorphic to $X(s, t, s-r)$. The existence of this isomorphism can be also obtained from the following statement.

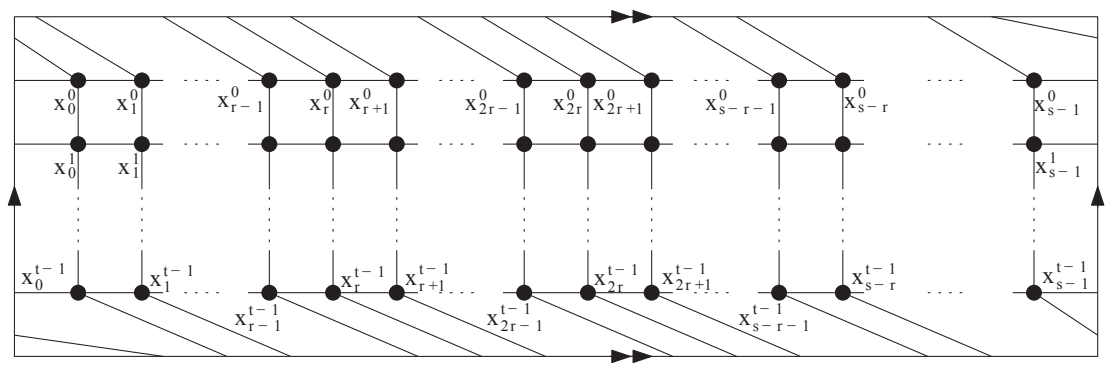

Figure 1: The graph $X(s, t, r)$ is embedded into torus with quadrilateral faces; it has a blue and red 2-factorization: the vertical and diagonal edges form the blue 2 -factor, the horizontal edges form the red 2 -factor. 
Proposition 4.1. Let $\operatorname{Cay}\left(\Gamma,\left\{ \pm \gamma_{1}, \pm \gamma_{2}\right\}\right)$ be a connected Cayley multigraph of degree 4, where $\Gamma$ is an abelian group, $o\left(\gamma_{1}\right)=s$ and $|\Gamma| / s=t$. Then a $\gamma_{2}=r \gamma_{1}$ for some integer $r$, $0 \leq r \leq s-1$, if and only if $a=t$. Consequently, $\operatorname{Cay}\left(\Gamma,\left\{ \pm \gamma_{1}, \pm \gamma_{2}\right\}\right)$ can be represented as the graph $X(s, t, r)$ or $X(s, t, s-r)$.

Proof. We show that $G_{1}=\operatorname{Cay}\left(\Gamma,\left\{ \pm \gamma_{1}, \pm \gamma_{2}\right\}\right)$ and $G_{2}=X(s, t, r)$ are isomorphic. Since $\gamma_{1}$ and $\gamma_{2}$ are generators of $\Gamma$, the elements of $\Gamma$ can be written in the form $i \gamma_{2}+j \gamma_{1}$, where $i \gamma_{2} \in\left\langle\gamma_{2}\right\rangle, j \gamma_{1} \in\left\langle\gamma_{1}\right\rangle$. Hence we can describe the elements of $\Gamma$ by the left cosets of the subgroup $\left\langle\gamma_{1}\right\rangle$ in $\Gamma$. By this representation, we can see that the endvertices of an edge $\left[x, x \pm \gamma_{1}\right]$ of $\operatorname{Cay}\left(\Gamma,\left\{ \pm \gamma_{1}, \pm \gamma_{2}\right\}\right)$ belong to the same left coset of $\left\langle\gamma_{1}\right\rangle$ in $\Gamma$; the endvertices of an edge $\left[x, x \pm \gamma_{2}\right]$ belong to distinct left cosets of $\left\langle\gamma_{1}\right\rangle$ in $\Gamma$. Therefore, $a \gamma_{2}=r \gamma_{1} \in\left\langle\gamma_{1}\right\rangle$ if and only if $a=t$, since $\operatorname{Cay}\left(\Gamma,\left\{ \pm \gamma_{1}, \pm \gamma_{2}\right\}\right)$ is connected and $\left|\Gamma /\left\langle\gamma_{1}\right\rangle\right|=t$. Hence we can set $V\left(G_{1}\right)=\left\{i \gamma_{2}+j \gamma_{1}: 0 \leq i \leq t-1,0 \leq j \leq s-1\right\}$ and $E\left(G_{1}\right)=\left\{\left[i \gamma_{2}+j \gamma_{1},(i+1) \gamma_{2}+j \gamma_{1}\right],\left[i \gamma_{2}+j \gamma_{1}, i \gamma_{2}+(j+1) \gamma_{1}\right]: 0 \leq i \leq t-1,0 \leq j \leq\right.$ $s-1\}$. The map $\varphi: V\left(G_{1}\right) \rightarrow V\left(G_{2}\right)$ defined by $\varphi\left(i \gamma_{2}+j \gamma_{1}\right)=x_{j}^{i}$ is a bijection between $V\left(G_{1}\right)$ and $V\left(G_{2}\right)$. Moreover, if $v_{1}, v_{2}$ are adjacent vertices of $G_{1}$, that is, $v_{1}=i \gamma_{2}+j \gamma_{1}$ and $v_{2}=(i+1) \gamma_{2}+j \gamma_{1}$ (or $\left.v_{2}=i \gamma_{2}+(j+1) \gamma_{1}\right)$, then $\varphi\left(v_{1}\right)=x_{j}^{i}, \varphi\left(v_{2}\right)=x_{j}^{i+1}$ (or $\varphi\left(v_{2}\right)=x_{j+1}^{i}$ ) are adjacent vertices of $G_{2}$. In particular, if $v_{1}=(t-1) \gamma_{2}+j \gamma_{1}$ and $v_{2}=t \gamma_{2}+j \gamma_{1}=r \gamma_{1}+j \gamma_{1}=(r+j) \gamma_{1}$, then $\varphi\left(v_{1}\right)=x_{j}^{t-1}, \varphi\left(v_{2}\right)=x_{r+j}^{0}$ are adjacent vertices of $G_{2}$. It is thus proved that $\varphi$ is an isomorphism between $G_{1}$ and $G_{2}$. If we replace the element $\gamma_{1}$ by its inverse $-\gamma_{1}$, then $G_{1}$ is the graph $X(s, t, s-r)$.

In what follows, we show that for $s, t \geq 1$ there exists a Cayley multigraph on a suitable abelian group that satisfies Proposition 4.1, that is, for every $s, t \geq 1$ the graph $X(s, t, r)$ can be represented as a Cayley multigraph. The proof is particularly easy when $t=1$; $r=0$; or $s=2$. For these cases, the following holds.

Proposition 4.2. The graph $X(s, 1, r)$, with $s \geq 1,0 \leq r \leq s-1$, is the circulant multigraph $\operatorname{Cir}(s ; \pm 1, \pm r)$. The graph $X(s, t, 0)$, with $s, t \geq 1$, is the Cayley multigraph $\operatorname{Cay}\left(\mathbb{Z}_{s} \times \mathbb{Z}_{t},\{ \pm(1,0), \pm(0,1)\}\right)$. The graph $X(2, t, 1)$, with $t \geq 1$, is the circulant multigraph $\operatorname{Cir}(2 t ; \pm t, \pm 1)$.

Proof. For the graph $X(s, 1, r)$ we apply Proposition 4.1 with $\Gamma=\mathbb{Z}_{s t}, \gamma_{1}=1, \gamma_{2}=$ $r$. For the graph $X(s, t, 0)$ we apply Proposition 4.1 with $\Gamma=\mathbb{Z}_{s} \times \mathbb{Z}_{t}, \gamma_{1}=(1,0)$, $\gamma_{2}=(0,1)$. For the graph $X(2, t, 1)$ we apply Proposition 4.1 with $\Gamma=\mathbb{Z}_{2 t}, \gamma_{1}=t$, $\gamma_{2}=1$.

The following lemmas concern the graph $X(s, t, r)$ with $s \geq 3, t \geq 2$ and $0<r \leq$ $s-1$. They will be used in the proof of Proposition 4.5.

Lemma 4.3. Let $a>1$ be an integer and let $b \geq 1$ be a divisor of $a$. Let $\left\{[c]_{b}: 0 \leq c \leq\right.$ $b-1\}$ be the residue classes modulo $b$. Every equivalence class $[c]_{b}$ whose representative $c$ is coprime with b contains at least one integer $h, 1 \leq h \leq a-1$, such that $\operatorname{gcd}(a, h)=1$.

Proof. The assertion is true if $b=a$ (we set $h=c$ ). We consider $b<a$. Let $[c]_{b}$ be an equivalence class modulo $b$ with $1 \leq c \leq b-1$ and $\operatorname{gcd}(c, b)=1$. If $c$ is coprime with $a$, then we set $h=c$ and the assertion follows. We consider the case $\operatorname{gcd}(c, a) \neq 1$. We denote by $\mathcal{P}$ the set of distinct prime numbers dividing $a$. We denote by $\mathcal{P}_{b}$ (respectively, by $\mathcal{P}_{c}$ ) the subset of $\mathcal{P}$ containing the prime numbers dividing $b$ (respectively, $c$ ). Since $b$ is a divisor of $a($ respectively, $\operatorname{gcd}(c, a) \neq 1)$, the set $\mathcal{P}_{b}$ (respectively, $\mathcal{P}_{c}$ ) is non-empty. 
Since $c$ and $b$ are coprime, the subsets $\mathcal{P}_{b}, \mathcal{P}_{c}$ are disjoint. We set $\mathcal{P}^{\prime}=\mathcal{P} \backslash\left(\mathcal{P}_{b} \cup \mathcal{P}_{c}\right)$. The set $\mathcal{P}^{\prime}$ might be empty. We denote by $\omega$ the product of the prime numbers in $\mathcal{P}^{\prime}$ (if $\mathcal{P}^{\prime}$ is empty, then we set $\omega=1$ ) and consider the integer $h=c+\omega b \in[c]_{b}$. We show that $h<a$. Note that $\omega \leq a /(2 b)$. More specifically, $(a / b) \geq\left(\prod_{p \in \mathcal{P}_{c}} p\right) \cdot \omega \geq 2 \omega$, whence $\omega \leq a /(2 b)$. Hence $h=c+\omega b \leq c+(a / 2)<a$, since $c<b$ and $b \leq(a / 2)$. One can easily verify that $\operatorname{gcd}(h, a)=\operatorname{gcd}(c+\omega b, a)=1$, since no prime number in $\mathcal{P}=\mathcal{P}^{\prime} \cup \mathcal{P}_{b} \cup \mathcal{P}_{c}$ can divide $c+\omega b$. The assertion follows.

Lemma 4.4. Let $s \geq 3, t \geq 2$ and $\mathbb{Z}_{s t / d_{1}}$ be the cyclic group of integers modulo $s t / d_{1}$, where $d_{1} \geq 1$ is a divisor of $d=\operatorname{gcd}(s, t)$. Let $\left\langle t / d_{1}\right\rangle$ be the cyclic subgroup of $\mathbb{Z}_{s t / d_{1}}$ generated by the integer $t / d_{1}$ and let $x+\left\langle t / d_{1}\right\rangle, y+\left\langle t / d_{1}\right\rangle$ be left cosets of $\left\langle t / d_{1}\right\rangle$ in $\mathbb{Z}_{s t / d_{1}}$. If $x, y \in \mathbb{Z}_{s t / d_{1}}$ are congruent modulo $d / d_{1}$, then $t\left(x+\left\langle t / d_{1}\right\rangle\right)=\left\{t x+\mu t^{2} / d_{1}\right.$ : $0 \leq \mu \leq s-1\}$ and $t\left(y+\left\langle t / d_{1}\right\rangle\right)=\left\{t y+\mu^{\prime} t^{2} / d_{1}: 0 \leq \mu^{\prime} \leq s-1\right\}$ represent the same subset of $\mathbb{Z}_{s t / d_{1}}$.

Proof. Set $x=y+\lambda d / d_{1}$ with $\lambda \in \mathbb{Z}$ and $t=s m^{\prime}+m$ with $m^{\prime} \in \mathbb{Z}$ and $0 \leq m \leq s-1$. Since $\operatorname{gcd}(s, t)=d$, then also $\operatorname{gcd}(s, m)=d$. Hence the integers $d t / d_{1}, m t / d_{1} \in \mathbb{Z}_{s t / d_{1}}$ generate the same cyclic subgroup of $\left\langle t / d_{1}\right\rangle$ of order $s / d$. Since $t^{2} / d_{1}=\left(s m^{\prime}+m\right) t / d_{1} \equiv$ $m t / d_{1}\left(\bmod s t / d_{1}\right)$, each set $t\left(x+\left\langle t / d_{1}\right\rangle\right), t\left(y+\left\langle t / d_{1}\right\rangle\right)$ consists of exactly $s / d$ distinct elements of $\mathbb{Z}_{s t / d_{1}}$, namely, $t\left(x+\left\langle t / d_{1}\right\rangle\right)=\left\{t x+\mu m t / d_{1}: 1 \leq \mu \leq s / d\right\}, t(y+$ $\left.\left\langle t / d_{1}\right\rangle\right)=\left\{t y+\mu^{\prime} m t / d_{1}: 1 \leq \mu^{\prime} \leq s / d\right\}$. Therefore, to prove that $t\left(x+\left\langle t / d_{1}\right\rangle\right)=$ $t\left(y+\left\langle t / d_{1}\right\rangle\right)$, it suffices to show that every element of $t\left(x+\left\langle t / d_{1}\right\rangle\right)$ is contained in $t(y+$ $\left.\left\langle t / d_{1}\right\rangle\right)$. Consider $t x+\mu m t / d_{1} \in t\left(x+\left\langle t / d_{1}\right\rangle\right)$. Since $x=y+\lambda d / d_{1}$, we can write $t x+\mu m t / d_{1}=t\left(y+\lambda d / d_{1}\right)+\mu m t / d_{1}$, whence $t x+\mu m t / d_{1}=t y+\lambda d t / d_{1}+\mu m t / d_{1}$. Since $\left\langle d t / d_{1}\right\rangle=\left\langle m t / d_{1}\right\rangle$, we can set $\lambda d t / d_{1}+\mu m t / d_{1} \equiv \mu^{\prime} m t / d_{1}\left(\bmod s t / d_{1}\right)$, with $0 \leq \mu^{\prime} \leq s / d$. Hence $t x+\mu m t / d_{1} \equiv t y+\mu^{\prime} m t / d_{1}\left(\bmod s t / d_{1}\right)$, that is, $t x+\mu m t / d_{1} \in$ $t\left(y+\left\langle t / d_{1}\right\rangle\right)$. The assertion follows.

Proposition 4.5. Let $s \geq 3, t \geq 2,0<r \leq s-1$, with $\operatorname{gcd}(s, t, r)=d_{1}$. The cyclic group $\mathbb{Z}_{s t / d_{1}}$ of integers modulo st $/ d_{1}$ contains an integer $k$ such that $\operatorname{gcd}(k, t)=1$ and $k \equiv r / d_{1}\left(\bmod s / d_{1}\right)$. The graph $X(s, t, r)$ can be represented as the Cayley graph $\operatorname{Cay}\left(\mathbb{Z}_{s t / d 1} \times \mathbb{Z}_{d_{1}},\left\{ \pm\left(t / d_{1}, 1\right), \pm(k, 0)\right\}\right)$. In particular, if $d_{1}=1$ then $X(s, t, r)$ can be represented as the circulant graph $\operatorname{Cir}(s t ; \pm t, \pm k)$.

Proof. Set $d=\operatorname{gcd}(s, t)$. Since $\operatorname{gcd}(s, t, r)=d_{1}$, the integer $r / d_{1}$ is coprime with $\operatorname{gcd}\left(s / d_{1}, t / d_{1}\right)=d / d_{1}$. Hence, $r / d_{1}$ belongs to an equivalence class $[c]_{d / d_{1}}$ whose representative is coprime with $d / d_{1}$. By Lemma 4.3 , the class $[c]_{d / d_{1}}$ contains an integer $h, 1 \leq h<t$, such that $\operatorname{gcd}(h, t)=1$. Consider the cyclic group $\mathbb{Z}_{s t / d_{1}}$. Since $r / d_{1}<s, h<t$, the integer $r / d_{1}$ and $h$ belong to $\mathbb{Z}_{s t / d_{1}}$. Hence we can apply Lemma 4.4 with $x=r / d_{1}, y=h$ and find that $t\left(r / d_{1}+\left\langle t / d_{1}\right\rangle\right)=t\left(h+\left\langle t / d_{1}\right\rangle\right)$, that is, there exists an integer $k \in h+\left\langle t / d_{1}\right\rangle$ such that $t k \equiv r t / d_{1}\left(\bmod s t / d_{1}\right)$. The integer $k$ is coprime with $t$, since $\operatorname{gcd}(h, t)=1$. The assertion follows from Proposition 4.1, by setting $\Gamma=\mathbb{Z}_{s t / d_{1}} \times \mathbb{Z}_{d_{1}}, \gamma_{1}=\left(t / d_{1}, 1\right), \gamma_{2}=(k, 0)$. Note: if $d_{1}=1$, then $\Gamma$ is the cyclic group of order st, $\gamma_{1}=t, \gamma_{2}=k$ and $X(s, t, r)$ is the circulant graph $\operatorname{Cir}(s t ; \pm t, \pm k)$.

The result that follows is based on a well-known consequence of the Chinese Remainder Theorem. More specifically, it is known that if $a, b$, and $n$ are positive integers, with $\operatorname{gcd}(a, n)=c \geq 1$, then the equation $a x \equiv b(\bmod n)$ admits a solution if and only if $c$ is a divisor of $b$ and in this case $x \equiv(a / c)^{-1}(b / c)(\bmod n / c)$ is a solution to the equation. The following holds. 
Proposition 4.6. Let $s, t \geq 1,0 \leq r \leq s-1$ and $d_{1}=\operatorname{gcd}(s, t, r)$. If $r \neq 0$, then there exists an integer $k, 0<k<s t / d_{1}$, such that $\operatorname{gcd}(k, t)=1$ and $k \equiv r / d_{1}\left(\bmod s / d_{1}\right)$. The graph $X(s, t, r)$, with $r \neq 0$, is isomorphic to the $\operatorname{graph} X(s t / \operatorname{gcd}(s, r), \operatorname{gcd}(s, r)$, $\left.r^{\prime}\right)$, where $r^{\prime} \equiv \pm t\left(k d_{1} / \operatorname{gcd}(s, r)\right)^{-1}(\bmod s t / \operatorname{gcd}(s, r))$. The graph $X(s, t, 0)$ is isomorphic to the graph $X(t, s, 0)$.

Proof. We prove the assertion for $s \geq 3, t \geq 2$ and $0<r \leq s-1$. The existence of the integer $k$ follows from Proposition 4.5. By the same proposition, we can represent the graph $X(s, t, r)$ as $\operatorname{Cay}\left(\mathbb{Z}_{s t / d 1} \times \mathbb{Z}_{d_{1}},\left\{ \pm\left(t / d_{1}, 1\right), \pm(k, 0)\right\}\right)$. We apply Proposition 4.1 by setting $\Gamma=\mathbb{Z}_{s t / d 1} \times \mathbb{Z}_{d_{1}}, \gamma_{1}=(k, 0)$ and $\gamma_{2}=\left(t / d_{1}, 1\right)$. Note that $\operatorname{gcd}\left(s t / d_{1}, k\right)=$ $\operatorname{gcd}\left(s / d_{1}, k\right)=\operatorname{gcd}(s, r) / d_{1}$, as $k$ is coprime with $t$ and $k \equiv r / d_{1}\left(\bmod s / d_{1}\right)$. Whence the element $(k, 0)$ has order

$$
s^{\prime}=s t /\left(d_{1} \operatorname{gcd}\left(s t / d_{1}, k\right)\right)=s t / \operatorname{gcd}(s, r) \text { and } t^{\prime}=|\Gamma /\langle(k, 0)\rangle|=\operatorname{gcd}(s, r) .
$$

By Proposition 4.1, $\operatorname{gcd}(s, r)\left(t / d_{1}, 1\right)=r^{\prime}(k, 0)$ for some integer $r^{\prime}, 1 \leq r^{\prime} \leq s t / \operatorname{gcd}(s$, $r)$. The integer $r^{\prime}$ is a solution to the equation $\operatorname{gcd}(s, r)\left(t / d_{1}\right) \equiv r^{\prime} k\left(\bmod s t / d_{1}\right)$. By the Chinese Remainder Theorem, $r^{\prime} \equiv t\left(k d_{1} / \operatorname{gcd}(s, r)\right)^{-1}(\bmod s t / \operatorname{gcd}(s, r))$. An easy calculation shows that $s^{\prime}-r^{\prime} \equiv-t\left(k d_{1} / \operatorname{gcd}(s, r)\right)^{-1}(\bmod s t / \operatorname{gcd}(s, r))$. It is straightforward to see that $X(s, t, r)$ and $X\left(s^{\prime}, t^{\prime}, r^{\prime}\right), X\left(s^{\prime}, t^{\prime}, s^{\prime}-r^{\prime}\right)$ are isomorphic. Hence the assertion follows. For the remaining values of $s, t, r$, we represent the graph $X(s, t, r)$ as the Cayley multigraph in Proposition 4.2 and use Proposition 4.1. Note: if $r \neq 0$, then $k=r$; if $r=0$, then set $\gamma_{1}=(0,1), \gamma_{2}=(1,0)$ and apply Proposition 4.1.

\subsection{Fundamental 2-factorization of $X(s, t, r)$}

From the definition of $X(s, t, r)$ one can see that the horizontal edges form a 2 -factor (the red 2-factor) whose complementary 2 -factor in $X(s, t, r)$ is given by the vertical and diagonal edges (the blue 2-factor). We say that the red and blue 2-factor constitute the fundamental 2-factorization of $X(s, t, r)$. A graph $X(s, t, r)$ can be represented as a Cayley multigraph $\operatorname{Cay}\left(\Gamma,\left\{ \pm \gamma_{1}, \pm \gamma_{2}\right\}\right)$, where $\Gamma$ and $\left\{ \pm \gamma_{1}, \pm \gamma_{2}\right\}$ can be defined as in Proposition 4.2 or 4.5 . From the proof of the propositions, one can see that the set of horizontal edges of $X(s, t, r)$ is the set $\left\{\left[x, x \pm \gamma_{1}\right]: x \in \Gamma\right\}$, the set of vertical and diagonal edges is the set $\left\{\left[x, x \pm \gamma_{2}\right]: x \in \Gamma\right\}$. The edges in $\left\{\left[x, x \pm \gamma_{1}\right]: x \in \Gamma\right\}$ will be called the $\gamma_{1}$-edges and the edges in the set $\left\{\left[x, x \pm \gamma_{2}\right]: x \in \Gamma\right\}$ will be called the $\gamma_{2}$-edges. The following result holds.

Proposition 4.7. The red 2-factor of $X(s, t, r)$ has exactly $t$ cycles of length s consisting of $\gamma_{1}$-edges. The blue 2 -factor of $X(s, t, r)$ has exactly $\operatorname{gcd}(s, r)$ cycles of length st $/ \operatorname{gcd}(s, r)$ consisting of $\gamma_{2}$-edges.

Proof. It is straightforward to see that the red 2-factor has $t$ horizontal cycles of length $s$ (if $s=1$, then each cycle is a loop; if $s=2$, then each cycle is a dipole with 2 parallel edges). By the previous remarks, each cycle consists of $\gamma_{1}$-edges. The blue 2 -factor of $X(s, t, r)$ corresponds to the red 2-factor of the graph $X\left(s t / \operatorname{gcd}(s, r), \operatorname{gcd}(s, r), r^{\prime}\right)$ in Proposition 4.6. Hence it has $\operatorname{gcd}(s, r)$ cycles of length $s t / \operatorname{gcd}(s, r)$ consisting of $\gamma_{2}$-edges.

\subsection{Isomorphisms between $X(s, t, r)$ graphs}

We now ask when two graphs $X(s, t, r)$ and $X\left(s^{\prime}, t^{\prime}, r^{\prime}\right)$ are isomorphic. Our question is connected to the following well-known problem [7, 14]. Given two isomorphic Cayley 
multigraphs $\operatorname{Cay}(\Gamma, S), \operatorname{Cay}\left(\Gamma^{\prime}, S^{\prime}\right)$ or, equivalently, given two Cayley representations $(\Gamma, S),\left(\Gamma^{\prime}, S^{\prime}\right)$ of the same multigraph, determine whether $(\Gamma, S)$ and $\left(\Gamma^{\prime}, S^{\prime}\right)$ are equivalent. We recall that two Cayley representations of the same multigraph are said to be equivalent if there exists a permutation on the vertex-set of the multigraph that induces an isomorphism from the group $\Gamma$ to the group $\Gamma^{\prime}$ and sends $S$ onto $S^{\prime}$. Two Cayley representations $(\Gamma, S),\left(\Gamma, S^{\prime}\right)$ are equivalent if and only if there exists an automorphism $\sigma$ of the group $\Gamma$ that sends $S$ onto $S^{\prime}$ (see [14]). The automorphism $\sigma$ is called a CI-isomorphism (CI stands for Cayley Isomorphism). Ádám [1] considered this problem for circulant graphs and formulated a well-known conjecture which was disproved in [9]. He conjectured that two circulant graphs $\operatorname{Cir}(n ; S), \operatorname{Cir}\left(n ; S^{\prime}\right)$ are isomorphic if and only if there exists an integer $m^{\prime} \in \mathbb{Z}_{n}, \operatorname{gcd}\left(m^{\prime}, n\right)=1$, such that $S^{\prime}=\left\{m^{\prime} x: x \in S\right\}$. Even though the conjecture was disproved, there are some circulant graphs for which it holds (see for instance [16]). In [7] the problem is studied for Cayley multigraphs of degree 4 which are associated to abelian groups. The results in [7] are described in terms of Ádám isomorphisms. An Ádám isomorphism from $\operatorname{Cay}(\Gamma, S)$ to $\operatorname{Cay}\left(\Gamma^{\prime}, S^{\prime}\right)$ is an isomorphism obtained from a permutation on the vertex-set of $\operatorname{Cay}(\Gamma, S)$, that makes $(\Gamma, S),\left(\Gamma^{\prime}, S^{\prime}\right)$ equivalent, and an automorphism of the graph $\operatorname{Cay}\left(\Gamma^{\prime}, S^{\prime}\right)$. By the definition of equivalent Cayley representations, the existence of an Ádám isomorphism means that the groups $\Gamma, \Gamma^{\prime}$ are isomorphic and there exists an isomorphism between the groups that sends $S$ onto $S^{\prime}$. An Ádám isomorphism between $\operatorname{Cay}(\Gamma, S)$ and $\operatorname{Cay}\left(\Gamma, S^{\prime}\right)$ is a CI-isomorphism. Since the graphs $X(s, t, r)$ can be represented as Cayley multigraphs, we can extend the notion of Ádám isomorphism to the graphs $X(s, t, r)$. We will say that the graphs $X(s, t, r), X\left(s^{\prime}, t^{\prime}, r^{\prime}\right)$ are Ádám isomorphic if the corresponding Cayley multigraphs $\operatorname{Cay}\left(\Gamma,\left\{ \pm \gamma_{1}, \pm \gamma_{2}\right\}\right), \operatorname{Cay}\left(\Gamma^{\prime},\left\{ \pm \gamma_{1}^{\prime}, \pm \gamma_{2}^{\prime}\right\}\right)$, respectively, are Ádám isomorphic $\left(\operatorname{Cay}\left(\Gamma,\left\{ \pm \gamma_{1}, \pm \gamma_{2}\right\}\right), \operatorname{Cay}\left(\Gamma^{\prime},\left\{ \pm \gamma_{1}^{\prime}, \pm \gamma_{2}^{\prime}\right\}\right)\right.$ are described in Proposition 4.2 or 4.5 ). The following statements hold.

Proposition 4.8. Every Ádám isomorphism between the graphs $X(s, t, r), X\left(s^{\prime}, t^{\prime}, r^{\prime}\right)$ sends the fundamental 2-factorization of $X(s, t, r)$ onto the fundamental 2-factorization of $X\left(s^{\prime}, t^{\prime}, r^{\prime}\right)$.

Proof. An Ádám isomorphism between the graphs $\operatorname{Cay}\left(\Gamma,\left\{ \pm \gamma_{1}, \pm \gamma_{2}\right\}\right), \operatorname{Cay}\left(\Gamma^{\prime},\left\{ \pm \gamma_{1}^{\prime}\right.\right.$, $\left.\left.\pm \gamma_{2}^{\prime}\right\}\right)$ sends a $\gamma_{i}$-edge, $i=1,2$, of $\operatorname{Cay}\left(\Gamma,\left\{ \pm \gamma_{1}, \pm \gamma_{2}\right\}\right)$ onto a $\tau\left(\gamma_{i}\right)$-edge of $\operatorname{Cay}\left(\Gamma^{\prime}\right.$, $\left.\left\{ \pm \gamma_{1}^{\prime}, \pm \gamma_{2}^{\prime}\right\}\right)$, where $\tau\left(\gamma_{i}\right) \in\left\{ \pm \gamma_{1}^{\prime}, \pm \gamma_{2}^{\prime}\right\}$. Since Proposition 4.7 holds, every Ádám isomorphism sends the red (respectively, the blue) 2-factor of $X(s, t, r)$ onto the red (respectively, the blue) 2 -factor of $X\left(s^{\prime}, t^{\prime}, r^{\prime}\right)$ or vice versa.

Proposition 4.9. Let $s, t \geq 1,0 \leq r \leq s-1$ and $\operatorname{gcd}(s, t, r)=d_{1}$. If $r \neq 0$, then there exists an integer $k, 0<k<s t / d_{1}$, such that $\operatorname{gcd}(k, t)=1$ and $k \equiv r / d_{1}\left(\bmod s / d_{1}\right)$. The graphs $X(s, t, r)$, with $r \neq 0$, and $X\left(s^{\prime}, t^{\prime}, r^{\prime}\right)$ are Ádám isomorphic if and only if $s^{\prime}=$ $s, t^{\prime}=t, r^{\prime}=s-r$ or $s^{\prime}=s t / \operatorname{gcd}(s, r), t^{\prime}=\operatorname{gcd}(s, r)$ and $r^{\prime} \equiv \pm t\left(k d_{1} / \operatorname{gcd}(s, r)\right)^{-1}$ $(\bmod s t / \operatorname{gcd}(s, r))$. The graphs $X(s, t, 0)$, and $X\left(s^{\prime}, t^{\prime}, r^{\prime}\right)$ are Ádám isomorphic if and only if $s^{\prime}=t, t^{\prime}=s$ and $r^{\prime} \equiv 0(\bmod t)$.

Proof. We prove the assertion for $s \geq 3, t \geq 2$ and $r \neq 0$. The graph $X(s, t, r)$ is the Cayley graph $\operatorname{Cay}\left(\mathbb{Z}_{s t / d_{1}} \times \mathbb{Z}_{d_{1}},\left\{ \pm\left(t / d_{1}, 1\right), \pm(k, 0)\right\}\right)$, since Proposition 4.5 holds. By Proposition 4.2 or 4.5 , we can represent $X\left(s^{\prime}, t^{\prime}, r^{\prime}\right)$ as the Cayley multigraph $\operatorname{Cay}\left(\Gamma^{\prime}\right.$, $\left.\left\{ \pm \gamma_{1}^{\prime}, \pm \gamma_{2}^{\prime}\right\}\right)$. The graphs $X(s, t, r), X\left(s^{\prime}, t^{\prime}, r^{\prime}\right)$ are Ádám isomorphic if and only if there exists an isomorphism $\tau$ between the groups $\mathbb{Z}_{s t / d_{1}} \times \mathbb{Z}_{d_{1}}$ and $\Gamma^{\prime}$ that sends the set $\left\{ \pm\left(t / d_{1}, 1\right), \pm(k, 0)\right\}$ onto the set $\left\{ \pm \gamma_{1}^{\prime}, \pm \gamma_{2}^{\prime}\right\}$. Without loss of generality, we can set 
$\left\{ \pm \gamma_{1}^{\prime}\right\}$

$\left\{ \pm \tau\left(\left(t / d_{1}, 1\right)\right)\right\}$ and $\left\{ \pm \gamma_{2}^{\prime}\right\}=\{ \pm \tau((k, 0))\}$. By the existence of $\tau$ we can identify the group $\Gamma^{\prime}$ with the group $\mathbb{Z}_{s t / d_{1}} \times \mathbb{Z}_{d_{1}}$. Hence $\gamma_{1}^{\prime}$ and $\gamma_{2}^{\prime}$ are elements of $Z_{s t / d_{1}} \times \mathbb{Z}_{d_{1}}$ of order $s$ and $s t / \operatorname{gcd}(s, r)$, respectively, since $\left(t / d_{1}, 1\right)$ and $(k, 0)$ have order $s$ and $s t / \operatorname{gcd}(s, r)$, respectively (see the proof of Proposition 4.5 and 4.6). It is an easy matter to prove that an element $(a, b) \in \mathbb{Z}_{s t / d_{1}} \times \mathbb{Z}_{d_{1}}$ has order $o(a) \cdot o(b) / \operatorname{gcd}(o(a), o(b))=$ $s t /\left(d_{1} \operatorname{gcd}\left(s t / d_{1}, a\right)\right)$, since $d_{1}$ is a divisor of $s$ and $t$. Hence $(a, b)$ has order $s$ if and only if $\operatorname{gcd}\left(s t / d_{1}, a\right)=t / d_{1}$, that is, $(a, b)=\left(m^{\prime} t / d_{1}, b\right)$ where $m^{\prime} \in \mathbb{Z}_{s t / d_{1}}, \operatorname{gcd}\left(m^{\prime}, s\right)=$ $1, b$ is an arbitrary element of $\mathbb{Z}_{d_{1}}$. The element $(a, b)$ has order $s t / \operatorname{gcd}(s, r)$ if and only if $\operatorname{gcd}\left(s t / d_{1}, a\right)=\operatorname{gcd}(s, r) / d_{1}=\operatorname{gcd}\left(s / d_{1}, k\right)$, since $k$ is coprime with $t$ and $k \equiv r / d_{1}\left(\bmod s / d_{1}\right)$. Hence $\operatorname{Cay}\left(\Gamma^{\prime},\left\{ \pm \gamma_{1}^{\prime}, \pm \gamma_{2}^{\prime}\right\}\right)$ is a graph of type $\operatorname{Cay}\left(\mathbb{Z}_{s t / d_{1}} \times\right.$ $\left.\mathbb{Z}_{d_{1}},\left\{ \pm\left(m^{\prime} t / d_{1}, b\right), \pm\left(a, b^{\prime}\right)\right\}\right)$, where $\operatorname{gcd}\left(m^{\prime}, s\right)=1, \operatorname{gcd}\left(s t / d_{1}, a\right)=\operatorname{gcd}\left(s / d_{1}, k\right), b$ and $b^{\prime}$ are suitable elements of $\mathbb{Z}_{d_{1}}$. Note that $a$ is coprime with $t$ and the relation $t a \equiv$ $r m^{\prime} t / d_{1}\left(\bmod s t / d_{1}\right)$ holds, since $\tau$ is an isomorphism and $t k \equiv r t / d_{1}\left(\bmod s t / d_{1}\right)$. If we apply Proposition 4.1 to the graph $G_{1}=\operatorname{Cay}\left(\mathbb{Z}_{s t / d_{1}} \times \mathbb{Z}_{d_{1}},\left\{ \pm\left(m^{\prime} t / d_{1}, b\right), \pm\left(a, b^{\prime}\right)\right\}\right)$ by setting $\gamma_{1}=\left(m^{\prime} t / d_{1}, b\right)$ (or $\gamma_{1}=-\left(m^{\prime} t / d_{1}, b\right)$ ), then $G_{1}$ can be represented as the graph $X(s, t, r)$ or $X(s, t, s-r)$. The graph $X(s, t, r)$ is isomorphic to the graph $G_{2}=X\left(s^{\prime}, t^{\prime}, r^{\prime}\right)$, where $s^{\prime}=s t / \operatorname{gcd}(s, r), t^{\prime}=\operatorname{gcd}(s, r), r^{\prime} \equiv \pm t\left(k d_{1} / \operatorname{gcd}(s, r)\right)^{-1}$ $(\bmod s t / \operatorname{gcd}(s, r))$, since Proposition 4.6 holds. Hence $G_{1}$ is isomorphic to $G_{2}$. The isomorphism between $G_{1}$ and $G_{2}$ can be obtained also by applying Proposition 4.6 to the graph $G_{1}$. For the remaining values of $s, t, r$ we represent the graph $X(s, t, r)$ as the Cayley multigraph in Proposition 4.2 and apply the previous method.

The results that follow are based on the following theorem of [7].

Theorem 4.10 ([7]). Any two finite isomorphic connected undirected Cayley multigraphs of degree 4 coming from abelian groups are Ádám isomorphic, unless they are obtained with the groups and sets $\mathbb{Z}_{4 n},\{ \pm 1, \pm(2 n-1)\}$ and $\mathbb{Z}_{2 n} \times \mathbb{Z}_{2},\{ \pm(1,0), \pm(1,1)\}$.

By Theorem 4.10 the existence of an isomorphism between two Cayley multigraphs of degree 4 , that are associated to abelian groups, implies the existence of an Ádám isomorphism, unless they are the graphs $\operatorname{Cir}(4 n ; \pm 1, \pm(2 n-1))$ and $\operatorname{Cay}\left(\mathbb{Z}_{2 n} \times \mathbb{Z}_{2},\{ \pm(1,0)\right.$, $\pm(1,1)\})$. The following statements are consequences of Theorem 4.10.

Corollary 4.11. The graphs $X(4 n, 1,2 n-1)$ and $X\left(s^{\prime}, t^{\prime}, r^{\prime}\right)$ are isomorphic if and only if $s^{\prime}=4 n, t^{\prime}=1, r^{\prime}=2 n+1$ or $s^{\prime}=2 n, t^{\prime}=2, r^{\prime} \in\{2,2 n-2\}$. Moreover, there is no isomorphism between $X(4 n, 1,2 n-1)$ and $X(2 n, 2,2)$ that sends the fundamental 2 -factorization of $X(4 n, 1,2 n-1)$ onto the fundamental 2-factorization of $X(2 n, 2,2)$.

Proof. The graph $X(4 n, 1,2 n-1)$ is the graph $\operatorname{Cir}(4 n ; \pm 1, \pm(2 n-1))$ (see Proposition 4.2). By Theorem 4.10, the graphs $X(4 n, 1,2 n-1)$ and $X\left(s^{\prime}, t^{\prime}, r^{\prime}\right)$ could be Ádám isomorphic or not. If they are Ádám isomorphic, then $s^{\prime}=4 n, t^{\prime}=1, r^{\prime}=2 n+1$, since Proposition 4.9 holds. If they are not Ádám isomorphic, then $X\left(s^{\prime}, t^{\prime}, r^{\prime}\right)$ is the graph $\operatorname{Cay}\left(\mathbb{Z}_{2 n} \times \mathbb{Z}_{2},\{ \pm(1,0), \pm(1,1)\}\right.$ ) (see Theorem 4.10). Hence $s^{\prime}=2 n, t^{\prime}=2, r^{\prime} \in$ $\{2,2 n-2\}$ (see Proposition 4.5 and 4.9). The fundamental 2 -factorization of $X(4 n, 1,2 n-$ 1) consists of two Hamiltonian cycles, whereas the fundamental 2 -factorization of $X(2 n$, 2,2 ) consists of two 2 -factors whose connected components are two $2 n$-cycles (see Proposition 4.7). Therefore no isomorphism between $X(4 n, 1,2 n-1)$ and $X(2 n, 2,2)$ can send the fundamental 2-factorization of $X(4 n, 1,2 n-1)$ onto the fundamental 2-factorization of $X(2 n, 2,2)$. 
Proposition 4.12. Let $X(s, t, r), X\left(s^{\prime}, t^{\prime}, r^{\prime}\right)$ be non-isomorphic to $X(4 n, 1,2 n-1)$, $X(2 n, 2,2)$. Then $X(s, t, r)$ and $X\left(s^{\prime}, t^{\prime}, r^{\prime}\right)$ are isomorphic if and only if they are Ádám isomorphic, that is, if and only if the parameters $s^{\prime}, t^{\prime}, r^{\prime}$ satisfy Proposition 4.9.

Proof. The assertion follows from Theorem 4.10, and Proposition 4.9.

\section{Special cubic graphs arising from $X(s, t, r)$ graphs}

When we consider graphs $X(s, t, r)$ we assume we are given a fundamental 2-factorization. This, in turn, implies we may turn the graph $X(s, t, r)$ into a cubic one by appropriately splitting each vertex. We note in passing that the operation of vertex-splitting and its converse were successfully used in a different context in [20].

There are two complementary possibilities. Either $X(s, t, r)$ arises from an $I$-graph or not. We consider each case separately.

\subsection{I-graphs arising from $X(s, t, r)$}

In Theorem 3.3 we remarked that any special cubic graph with a blue and red 2-factorization gives rise to the associated quartic graph with a blue and red 2 -factorization. In Lemma 3.5 , we showed that a proper $I$-graph $I(n, p, q)$ is special and gives rise to the associated circulant graph $Q(n, p, q)$. The following holds.

Lemma 5.1. The circulant graph $\operatorname{Cir}(n ; p, q)=Q(n, p, q)$ arising from a connected $I$ graph $I(n, p, q)$ by contracting the spokes is the graph $X(s, t, r)$ with $t=\operatorname{gcd}(n, q)$, $s=n / t \geq 3$ and $r \equiv \pm p(q / t)^{-1}(\bmod s)$.

Proof. The result follows from Proposition 4.1 by setting $\Gamma=\mathbb{Z}_{n}, \gamma_{1}=q, \gamma_{2}=p$. Whence $t p=r q$ for some integer $r, 0 \leq r \leq s-1$, that is, $r$ is a solution to the equation $r(q / t) \equiv p(\bmod s)$. By the Chinese Remainder Theorem, $r \equiv p(q / t)^{-1}(\bmod s)$.

Theorem 5.2. The graph $X(s, t, r)$ arises from a connected I-graph by contracting the spokes if and only if $\operatorname{gcd}(s, t, r)=1$ and $(t, r) \neq(2,0)$ for odd values of $s$. In this case, the graph $X(s, t, r)$ together with its fundamental 2-factorization, is in one-to-one correspondence with the $I$-graph $I(s t, k, t)$, where $0<k<s t, \operatorname{gcd}(k, t)=1$ and $k \equiv r(\bmod s)$ (in particular, $k=s$ if $r=0$ ). If at least one of the integers $k, t, \operatorname{gcd}(s, r)$ is 1 , then $X(s, t, r)$ corresponds to a generalized Petersen graph.

Proof. Assume that $X(s, t, r)$ arises from the connected $I$-graph $I(n, p, q)$ by contracting the spokes. By Lemma 5.1, $t=\operatorname{gcd}(n, q), s=n / t \geq 3$ and $r(q / t) \equiv p(\bmod s)$. Whence $(t, r) \neq(2,0)$ if $s$ is odd, otherwise $p=0$ (which is not possible). We show that $\operatorname{gcd}(s, t, r)=1$. Suppose, on the contrary, that $\operatorname{gcd}(s, t, r)=d_{1} \neq 1$, then $d_{1}$ is a divisor of $\operatorname{gcd}(t, p)$ since $r(q / t) \equiv p(\bmod s)$. That yields a contradiction, since $\operatorname{gcd}(t, p)=1$ (see Proposition 3.4). Hence $\operatorname{gcd}(s, t, r)=1$.

Assume that $\operatorname{gcd}(s, t, r)=1$. We show that $X(s, t, r)$ arises from a connected $I$-graph by contracting the spokes. Since $\operatorname{gcd}(s, t, r)=1$, the graph $X(s, t, r)$ can be represented as the circulant graph $\operatorname{Cir}(s t ; \pm t, \pm k)$, where $0<k<s t, \operatorname{gcd}(t, k)=1$ and $k \equiv r(\bmod s)$ (see Proposition 4.5). If $r=0$, then we can set $k=s$, since Proposition 4.2 holds. The graph $I(s t, k, t)$ is connected and it gives rise to the graph $X(s, t, r)$, since Lemma 5.1 holds. By Theorem 3.3, the graph $X(s, t, r)$, together with its fundamental 2-factorization, is in one-to-one correspondence with the $I$-graph $I(s t, k, t)$. If $k=1$ or $t=1$, then 
$X(s, t, r)$ corresponds to a generalized Petersen graph (see [3]). If $\operatorname{gcd}(s, r)=1$ then $X(s, t, r)$ is isomorphic to $X\left(s t, 1, r^{\prime}\right)$ (see Proposition 4.9. By the previous remarks, the graph $X\left(s t, 1, r^{\prime}\right)$ corresponds to a generalized Petersen graph. The assertion follows.

It is straightforward to see that isomorphic $X(s, t, r)$ graphs give rise to isomorphic $I$ graphs and also the converse is true. By Corollary 4.11 and Proposition 4.12, the circulant graphs $X(s, t, r), X\left(s^{\prime}, t^{\prime}, r^{\prime}\right)$ are isomorphic if and only if they are Ádám-isomorphic, that is, there exists an automorphism of the cyclic group of order $s t=s^{\prime} t^{\prime}$ that sends the defining set of the circulant graph $X(s, t, r)$ onto the defining set of the circulant graph $X\left(s^{\prime}, t^{\prime}, r^{\prime}\right)$. This fact is equivalent to the results proved in [13] about the isomorphism between $I$-graphs.

\subsection{Special Generalized $I$-graphs}

In this section we consider the special cubic graphs that correspond to the graphs $X(s, t, r)$ with $\operatorname{gcd}(s, t, r) \neq 1$, according to the correspondence described in Theorem 3.3. By Proposition 5.2, these special cubic graphs do not belong to the family of connected $I$ graphs. By Theorem 3.3 and Definition 1, we can define a family of special cubic graphs containing the family of connected $I$-graphs as a subfamily. We call this family Special Generalized I-graphs. This family is not contained in the family of GI-graphs [6].

Let $s \geq 1, t \geq 1$ and $0 \leq r \leq s-1$. We define a Special Generalized I-graph $S G I(s t, s, t, r)$ as a cubic graph of order st with vertex-set $V=\left\{u_{i, j}, u_{i, j}^{\prime}: 0 \leq i \leq\right.$ $t-1,0 \leq j \leq s-1\}$ and edge-set $E=\left\{\left[u_{i, j}, u_{i, j+1}\right],\left[u_{i, j}, u_{i, j}^{\prime}\right]: 0 \leq i \leq t-1,0 \leq j \leq\right.$ $s-1\} \cup\left\{\left[u_{i, j}^{\prime}, u_{i+1, j}^{\prime}\right]: 0 \leq i \leq t-2,0 \leq j \leq s-1\right\} \cup\left\{\left[u_{t-1, j}^{\prime}, u_{0, j+r}^{\prime}\right]: 0 \leq j \leq s-1\right\}$ (the addition $j+1$ and $j+r$ are considered modulo $s$ ). For $s=1$ or $(t, r)=(1,0)$, a special generalized $I$-graph has loops. For $s=2$ or $(t, r)=(2,0)$, it has multiple edges. For the other values of $s, t, r$, it is a simple cubic graph. We say that a vertex $u_{i, j}$ (respectively, $u_{i j}^{\prime}$ ) is an outer vertex (respectively, an inner vertex). We say that an edge $\left[u_{i, j}, u_{i, j+1}\right]$ (respectively, $\left[u_{i, j}^{\prime}, u_{i+1, j}^{\prime}\right]$ ) is an outer edge (respectively, an inner edge). We say that an edge $\left[u_{i, j}, u_{i, j}^{\prime}\right]$ is a spoke. The spokes constitute the special 1 -factor. The graph arising from $S G I(s t, s, t, r)$ by contracting the spokes is the graph $X(s, t, r)$. The horizontal edges of $X(s, t, r)$ correspond to the outer edges of $S G I(s t, s, t, r)$, vertical and diagonal edges of $X(s, t, r)$ correspond to the inner edges of $S G I(s t, s, t, r)$. A generalization of the proof of Proposition 5.2 gives the following statement.

Proposition 5.3. Let $s \geq 1, t \geq 1,0 \leq r \leq s-1$ and $d_{1}=\operatorname{gcd}(s, t, r)$. The graph $X(s, t, r)$, together with its fundamental 2-factorization, is in one-to-one correspondence with the graph $S G I(s t, s, t, k)$ where $k=s$ if $r=0$, otherwise $0<k<s t / d_{1}$, $\operatorname{gcd}(k, t)=1$ and $k \equiv r / d_{1}\left(\bmod s / d_{1}\right)$.

By Corollary 4.11, the graphs $X(4 n, 1,2 n-1)$ and $X(2 n, 2,2)$ are isomorphic, but no isomorphism between them sends the fundamental 2-factorization of $X(4 n, 1,2 n-1)$ onto the fundamental 2 -factorization of $X(2 n, 2,2)$. This fact means that the application of Theorem 3.3 to the graphs $X(4 n, 1,2 n-1)$ and $X(2 n, 2,2)$ yields non-isomorphic special cubic graphs. As a matter of fact, Proposition 5.2 says that the graph $X(4 n, 1,2 n-1)$ is in one-to-one correspondence with a connected $I$-graph, whereas $X(2 n, 2,2)$ does not correspond to any $I$-graph. For instance, for $n=2$ the graph $X(8,1,3)$ is associated with the Möbius-Kantor graph of girth 6, [15, 17], while $X(4,2,2)$ arises from a graph of girth 4 (see Figure 2). 


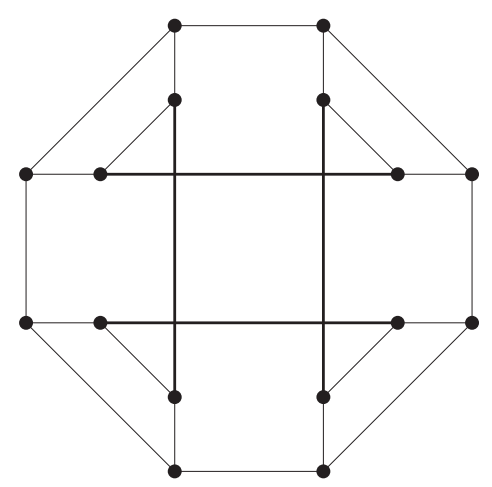

Figure 2: The cubic split $X(4,2,2)$ graph is $S G I(8,4,2,2)$. The thick edges represent the special 1-factor.

\section{Good Eulerian tours in $X(s, t, r)$ graphs}

In this section we construct good Eulerian subgraphs of $X(s, t, r)$. For each $X(s, t, r)$ we denote by $W(s, t, r)$ the constructed good Eulerian subgraph. By Proposition 3.2, a spanning Eulerian subgraph $W$ of $X(s, t, r)$ is admissible if and only if at each 2-valent vertex exactly one edge is horizontal. We consider $X(s, t, r)$ being embedded into the torus with quadrilateral faces. Hence any of its subgraphs may be viewed embedded in the same surface. A tour in $W$ may be regarded as as a straight-ahead walk (or SAW) on the surface [18]. A good Eulerian tour of $W$ is an Eulerian SAW that uses only allowed transitions, that is, the tour cannot switch from a horizontal to a vertical (or diagonal) edge when it visits a 4 -valent vertex of $W$. For instance, the graph $W$ in Figure 4(a) is an admissible subgraph of $X(5,4,3)$; the tour $\mathcal{E}=\left(x_{0}^{0}, x_{0}^{1}, x_{1}^{1}, x_{2}^{1}, x_{3}^{1}, x_{4}^{1}, x_{4}^{0}, x_{1}^{3}, x_{1}^{2}, x_{1}^{1}, x_{1}^{0}, x_{2}^{0}, x_{2}^{1}, x_{2}^{2}, x_{2}^{3}\right.$, $\left.x_{1}^{3}, x_{0}^{3}, x_{0}^{2}, x_{1}^{2}, x_{2}^{2}, x_{3}^{2}, x_{4}^{2}, x_{4}^{3}, x_{3}^{3}, x_{3}^{2}, x_{3}^{1}, x_{3}^{0}, x_{4}^{0}, x_{1}^{0}\right)$ is a good Eulerian SAW of $W$; hence $W$ is a good Eulerian subgraph of $X(5,4,3)$.

If we delete the diagonal edges in $X(s, t, r)$, we obtain a spanning subgraph that we denote by $X^{\prime}(s, t, r)$. Clearly $X^{\prime}(s, t, r)$ is the cartesian product of a cycle $C_{s}$ with a path $P_{t}$ embedded in the torus or cylinder. If we further delete an edge in $C_{s}$ we obtain a path $P_{s}$. We denote the cartesian product of $P_{s}$ and $P_{t}$ by $X^{\prime \prime}(s, t, r)$ and obtain a spanning subgraph of $X^{\prime}(s, t, r)$ and $X(s, t, r)$. In order to simplify the constructions, we will seek to find good Eulerian subgraphs in $X^{\prime}(s, t, r)$ or in $X^{\prime \prime}(s, t, r)$. In this case the resulting good Eulerian subgraph will be denoted by $W^{\prime}(s, t, r)$ and $W^{\prime \prime}(s, t, r)$, respectively. This simplification makes sense, since neither $X^{\prime}(s, t, r)$ nor $X^{\prime \prime}(s, t, r)$ depend on the parameter $r$. Hence any Eulerian subgraph $W^{\prime}(s, t, r)$ or $W^{\prime \prime}(s, t, r)$ is good for any $r$.

\subsection{Method of construction}

We give some lemmas that will be used in the construction of a good Eulerian subgraph $W(s, t, r)$. Given a graph $X(s, t, r)$, for every row index $i, 0 \leq i \leq t-1$, we denote by $V_{i}$ the set of vertical edges $V_{i}=\left\{\left[x_{j}^{i}, x_{j}^{i+1}\right]: 0 \leq j \leq s-1\right\}$. For every column index $j, 0 \leq$ $j \leq s-1$, we denote by $H_{j}$ the set of horizontal edges $H_{j}=\left\{\left[x_{j}^{i}, x_{j+1}^{i}\right]: 0 \leq i \leq t-1\right\}$.

Let $H$ be a subgraph of $X(s, t, r)$. We say that $H$ can be expanded vertically (from row 
i) if $\left|E(H) \cap V_{i}\right|=s-1$ or $s-2>0$ (for $s=3$ we require $\left|E(H) \cap V_{i}\right|=2$ ). We say that $H$ can be expanded horizontally (from column $j$ ) if $\left|E(H) \cap H_{j}\right|=t-1$ or $t-2>0$ (for $t=3$ we require $\left|E(H) \cap H_{j}\right|=2$ ). The following statements hold.

Lemma 6.1. Let $W\left(s, t_{1}, r\right)$ be a good Eulerian subgraph that can be expanded vertically. Then there exists a good Eulerian subgraph $W(s, t, r)$ for every $t \geq t_{1}, t \equiv t_{1}(\bmod 2)$.

Proof. We use the graph $W_{1}=W\left(s, t_{1}, r\right)$ to construct a good Eulerian subgraph $W(s, t, r)$. By the assumptions, $\left|E\left(W_{1}\right) \cap V_{i}\right|=s-1$ or $s-2$ for some row index $i$, $0 \leq i \leq t-1$. By the symmetry properties of the graph $X\left(s, t_{1}, r\right)$, we can cyclically permute its rows so that we can assume $0<i<t-1$. We treat separately the cases $\left|E\left(W_{1}\right) \cap V_{i}\right|=s-1$ and $\left|E\left(W_{1}\right) \cap V_{i}\right|=s-2$. Consider $\left|E\left(W_{1}\right) \cap V_{i}\right|=s-1$ and denote by $\left[x_{a}^{i}, x_{a}^{i+1}\right]$ the vertical edge of $V_{i}$ which is missing in $W_{1}$. We can cyclically permute the columns of $X\left(s, t_{1}, r\right)$ and assume $a=0$. We subdivide every vertical edge $\left[x_{j}^{i}, x_{j}^{i+1}\right]$, with $0<j \leq s-1$, by inserting two new vertices, namely, $y_{j}^{i}$ and $y_{j}^{i+1}$ such that $y_{j}^{i}$ is adjacent to $x_{j}^{i}$ and $y_{j}^{i+1}$ is adjacent to $x_{j}^{i+1}$, and we add two new vertices $y_{0}^{i+1}, y_{0}^{i}$ between $x_{0}^{i+1}$ and $x_{0}^{i}$ in column 0 . We now delete the edge $\left[y_{s-1}^{i}, y_{s-1}^{i+1}\right]$ and replace it with the path from $y_{s-1}^{i}$ to $y_{s-1}^{i+1}$ composed of the edges $\left[y_{j}^{i+1}, y_{j+1}^{i+1}\right],\left[y_{j}^{i}, y_{j+1}^{i}\right], 0 \leq j \leq s-2$, and $\left[y_{0}^{i}, y_{0}^{i+1}\right]$. The resulting graph is a good Eulerian subgraph $W\left(s, t_{1}+2, r\right)$. We can iterate the process and find a good Eulerian subgraph $W(s, t, r)$ for every $t \geq t_{1}, t \equiv t_{1}(\bmod 2)$. The case $\left|E\left(W_{1}\right) \cap V_{i}\right|=s-2$ can be treated analogously to the case $\left|E\left(W_{1}\right) \cap V_{i}\right|=s-1$. As an example, consider the graph $W^{\prime \prime}(6,5, r)$ in Figure 3. It can be expanded vertically from row 1 and it yields a good Eulerian subgraph $W^{\prime \prime}(6,7, r)$.
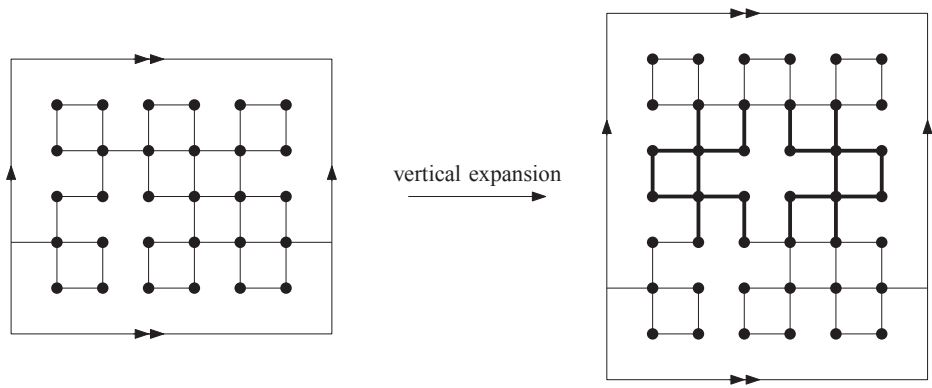

Figure 3: A vertical expansion of the good Eulerian subgraph $W^{\prime}(6,5, r)$ yields a good Eulerian subgraph $W(6,7, r)$.

In the following lemma we consider horizontal expansions. In this case we have to pay attention to the diagonal edges of $W(s, t, r)$, if any exist. If $\left[x_{j}^{t-1}, x_{j+r}^{0}\right]$, where $j+r$ is considered modulo $s$, is a diagonal edge of $W(s, t, r)$, then we can assume $j<j+r$, since we can cyclically permute the columns of $W(s, t, r)$. Therefore we can say that a diagonal edge $\left[x_{j}^{t-1}, x_{j+r}^{0}\right]$ crosses column $\ell$ if $j \leq \ell<j+r$.

Lemma 6.2. Let $W\left(s_{1}, t, r_{1}\right)$ be a good Eulerian subgraph that can be expanded horizontally from column $\ell$. If no diagonal edge of $W\left(s_{1}, t, r_{1}\right)$ crosses column $\ell$, then there exists a good Eulerian subgraph $W\left(s, t, r_{1}\right)$ for every $s \geq s_{1}, s \equiv s_{1}(\bmod 2)$. If every diagonal 
edge crosses column $\ell$, then there exists a good Eulerian subgraph $W\left(s_{1}+r-r_{1}, t, r\right)$ for every $r \geq r_{1}, r \equiv r_{1}(\bmod 2)$.

Proof. We apply the method described in Lemma 6.1 to the edges in $H_{\ell}$. If every diagonal edge of $W\left(s_{1}, t, r_{1}\right)$ crosses column $\ell$, then by subdividing the edges of $H_{\ell}$ we can shift of $r-r_{1}$ steps the diagonal edges of $W\left(s_{1}, t, r_{1}\right)$. If no diagonal edge of $W\left(s_{1}, t, r_{1}\right)$ crosses column $\ell$, then no diagonal edge is shifted. As an example, consider the graph $W(5,4,3)$ in Figure 4. If we expand horizontally the graph from column $\ell=0$, then no diagonal edge crosses column $\ell$ and we obtain a good Eulerian subgraph $W(7,4,3)$. If we expand horizontally the graph from column $\ell=2$, then every diagonal edge crosses column $\ell$ and we obtain a good Eulerian subgraph $W(7,4,5)$.

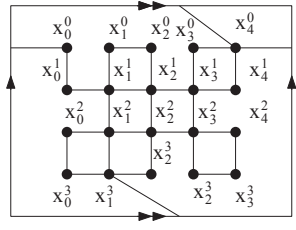

(a)

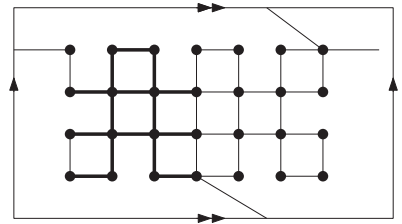

(b)

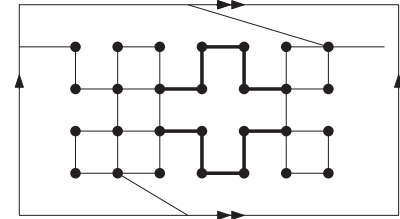

(c)

Figure 4: A good Eulerian subgraph: (a) $W(5,4,3)$; (b) $W(7,4,3)$; (c) $W(7,4,5)$. The graphs $W(7,4,3)$ and $W(7,4,5)$ are obtained from $W(5,4,3)$ by an horizontal expansion from column 0 and column 2 , respectively.

\subsection{Constructions of good Eulerian subgraphs.}

We apply the lemmas described in Section 6.1 to construct a good Eulerian subgraph $W(s, t, r)$. It is straightforward to see that the existence of loops in $X(s, t, r)$ excludes the existence of a good Eulerian subgraph $W(1, t, r)$ and $W(s, 1,0)$. Analogously, the existence of horizontal parallel edges in $X(2, t, r)$ excludes the existence of a good Eulerian subgraph $W(2, t, r)$ with $t$ odd and $W(2, t, 1)$ with $t$ even, $t>2$, (see Case 2 in the proof of Lemma 6.5 for a good Eulerian subgraph $W(2,2,1)$ and $W(2, t, 0)$ with $t$ even). Hence we can consider $s \geq 3$ and $(t, r) \neq(1,0)$. The following hold.

Proposition 6.3. The graph $X(s, 1, r), r \neq 0$, possesses a good Eulerian subgraph, unless $s=6 m+5$, with $m \geq 0$, and $r \in\{2, s-2,(s+1) / 2,(s-1) / 2\}$.

Proof. By Proposition 4.2, the graph $X(s, 1, r)$ can be represented as the circulant multigraph $\operatorname{Cir}(s t ; \pm 1, \pm r)$. By Proposition 5.2, the graph $X(s, 1, r)$ corresponds to the generalized Petersen graph $I(s, r, 1)$ or $G(s, r)$. In particular, the graph $X(6 m+5,1,2)$ corresponds to the generalized Petersen graph $G(6 m+5,2)$. Hence $X(s, 1, r)$ has a good Eulerian subgraph, unless it is isomorphic to $X(6 m+5,1,2)$, since Theorems 1.1 and 2.2 hold. By Proposition 4.12, the graphs that are isomorphic to $X(6 m+5,1,2)$ are $X\left(6 m+5,1, r^{\prime}\right)$, where $r^{\prime} \in\{2,6 m+3\}$ or $r^{\prime} \equiv \pm 2^{-1}(\bmod 6 m+5)$, that is, $r^{\prime} \in\{3 m+3,3 m+2\}$, since $r^{\prime}<6 m+5$.

We can construct a good Eulerian subgraph $W(s, 1, r), r \neq 0$, without using Theorem 1.1. More specifically, by Proposition 4.9 the graph $X(s, 1, r)$, with $r \neq 0$, is isomorphic 
to the $\operatorname{graph} X\left(s / \operatorname{gcd}(s, r), \operatorname{gcd}(s, r), r^{\prime}\right)$, where $r^{\prime} \equiv \pm r^{-1}(\bmod s)$. For $r \neq 0$ and $\operatorname{gcd}(s, r)>1$, a construction of a good Eulerian subgraph can be found in the proof of Lemma 6.5. We can also provide an ad hoc construction for the case $\operatorname{gcd}(s, r)=1$, but we prefer to omit this construction, since the existence of a good Eulerian subgraph $W(s, 1, r), r \neq 0$, is known (see Proposition 6.3) and the construction is based on the method of Lemma 6.5. We will show that the graph $X(6 m+5,1,2), m \geq 0$, has no good Eulerian subgraph, that is, the generalized Petersen graph is not Hamiltonian. The following statement is a consequence of Proposition 6.3 and it will be used in the proof of Lemma 6.5.

Proposition 6.4. The graph $X(s, t, r)$, with $s \geq 3, t>1$ and $\operatorname{gcd}(s, r)=1$ has a good Eulerian subgraph.

Proof. By Proposition 4.5, the graph $X(s, t, r)$ can be represented as the circulant graph $\operatorname{Cir}(s t ; \pm t, \pm k)$, where $\operatorname{gcd}(k, t)=1$ and $k \equiv r(\bmod s)$. By Proposition 4.9, the graph $X(s, t, r)$ is isomorphic to the graph $X\left(s t, 1, r^{\prime}\right)$, with $r^{\prime} \neq 0$, since $\operatorname{gcd}(s, r)=1$. If $s t \not \equiv 5(\bmod 6)$, then the assertion follows from Proposition 6.3 (see Proposition 4.9). Consider $s t \equiv 5(\bmod 6)$. We show that $X(s, t, r)$ is not isomorphic to $X(6 m+5,1,2)$, $m \geq 0$. Suppose, on the contrary, that $X(s, t, r)$ is isomorphic to $X(6 m+5,1,2)$. Then $X\left(s t, 1, r^{\prime}\right)=X\left(6 m+5,1, r^{\prime}\right)$, where $r^{\prime} \in\{2, s t-2,(s t+1) / 2,(s t-1) / 2\}$ (see Proposition 6.3). By Proposition 4.9, the integer $r^{\prime}$ satisfies the relation $r^{\prime} \equiv \pm t k^{-1}(\bmod s t)$. Whence $t$ is a divisor of $r^{\prime}$. That yields a contradiction, since $r^{\prime} \in\{2, s t-2,(s t+1) / 2$, $(s t-1) / 2\}$ and $t$ is coprime with the integers in $\{2, s t-2,(s t+1) / 2,(s t-1) / 2\}$.

Lemma 6.5. Let $s \geq 3, t \geq 2$ and $0 \leq r \leq s-1$. There exists a good Eulerian subgraph $W(s, t, r)$, unless $s$ is odd and $(t, r)=(2,0)$.

Proof. We treat separately the cases: $t=3 ; s, t$ even; $s$ even, $t$ odd, $t \geq 5 ; s$ odd, $t$ even; $s, t$ odd, $t \geq 5$. When we will speak of "vertical" and "horizontal" expansion we refer implicitly to Lemma 6.1 and 6.2, respectively.

Case 1: $t=3$. This case is treated in Section 8 , since it requires a lengthy description.

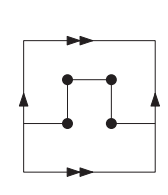

(a)

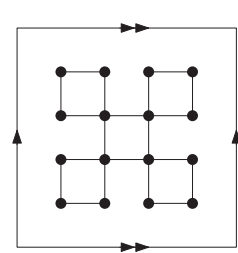

(b)

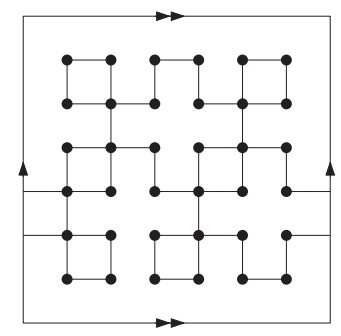

(c)

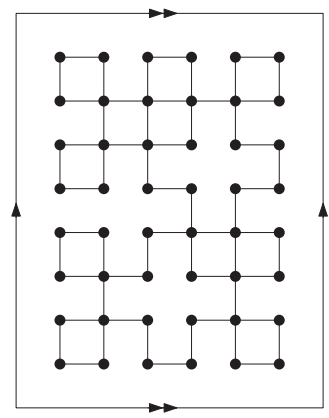

(d)

Figure 5: A good Eulerian subgraph: (a) $W^{\prime}(2,2, r)$; (b) $W^{\prime \prime}(4,4, r)$; (c) $W^{\prime}(6,6, r)$; (d) $W^{\prime \prime}(6,8, r)$. 
Case 2: $s$ even, $t$ even. The graph $W^{\prime \prime}(6,8, r)$ in Figure 5(d) can be expanded vertically from row 1 and horizontally from column 2. It yields a good Eulerian subgraph $W^{\prime \prime}(s, t, r)$ for every $s, t$ even $s \geq 6, t \geq 8$. It remains to construct a good Eulerian subgraph $W^{\prime \prime}(s, t, r)$ for $s \geq 6, t=2,4,6$ and $W^{\prime \prime}(4, t, r)$ for $t \geq 2, t$ even. The graph $W^{\prime}(2,2, r)$ in Figure $5($ a) can be expanded horizontally from column 0 or 1 . It yields a good Eulerian subgraph $W^{\prime}(s, 2, r)$ for every $s$ even, $s \geq 2$. We expand horizontally the graph $W^{\prime \prime}(4,4, r)$ in Figure 5(b) and obtain $W^{\prime \prime}(s, 4, r)$ for every $s$ even, $s \geq 4$. We rotate $W^{\prime \prime}(s, 4, r)$ by 90 degrees clockwise (around a vertex) and obtain a good Eulerian subgraph $W^{\prime \prime}(4, t, r)$ for every $t$ even, $t \geq 4$. We expand horizontally the graph $W^{\prime \prime}(6,6, r)$ in Figure 5(c) from column 3 and obtain $W^{\prime \prime}(s, 6, r)$ for every $s$ even, $s \geq 6$.

Case 3: $s$ even, $t$ odd, $t \geq 5$. The graph $W^{\prime}(6,5, r)$ in Figure 3 can be expanded vertically from row 2 and horizontally from column 3 . It yields a good Eulerian subgraph $W^{\prime}(s, t, r)$ for every $s$ even, $s \geq 6, t$ odd, $t \geq 5$. It remains to construct $W(4, t, r)$ with $t$ odd, $t \geq 5,0 \leq r \leq 3$. Since $X(4, t, r)$ is isomorphic to $X(4, t, 4-r)$, we can consider $0 \leq r \leq 2$. A good Eulerian subgraph for $W(4, t, 0), t$ odd, $t \geq 5$, can be obtained from $W(4,3,0)$ in Figure 6(a) by a vertical expansion from row 1 . The existence of a good Eulerian subgraph $W(4, t, 1)$ follows from Proposition 6.4. By Proposition 4.9, the graph $X(4, t, 2)$ is isomorphic to the graph $X\left(2 t, 2, r^{\prime}\right)$. By the results in Case 2, there exists a good Eulerian subgraph $W\left(2 t, 2, r^{\prime}\right)$.

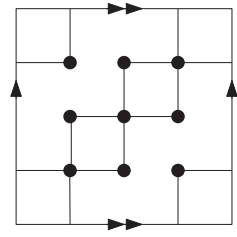

(a)

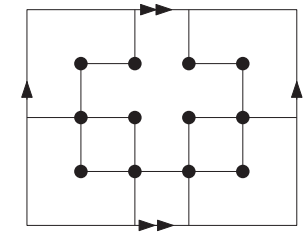

(b)

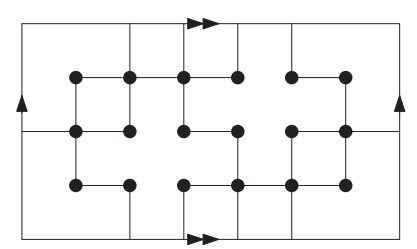

(c)

Figure 6: A good Eulerian subgraph: (a) $W(3,3,0)$; (b) $W(4,3,0)$; (c) $W(6,3,0)$.

Case 4: $s$ odd, $t$ even. By Proposition 4.9, the graph $X(s, t, r)$, with $r \neq 0$, is isomorphic to the graph $X\left(s t / \operatorname{gcd}(s, r), \operatorname{gcd}(s, r), r^{\prime}\right)$, with $r^{\prime} \neq 0$, or to $X(t, s, 0)$ if $r=0$. If $r \neq 0$ and $\operatorname{gcd}(s, r)=1$ or 3 , then the existence of a good Eulerian subgraph follows from Proposition 6.4 or from the results in Case 1, respectively. Note that $s t / \operatorname{gcd}(s, r) \geq 4$, since $t$ is even and $0<r \neq s-1$. Hence, for $\operatorname{gcd}(s, r) \geq 5$, the existence of a good Eulerian subgraph follows from Case 3. Consider $r=0$. There is no good Eulerian subgraph $W(s, 2,0)$, because of the existence of parallel vertical edges. Consider $t \geq 4$. As remarked, the graph $X(s, t, 0)$ is isomorphic to the graph $X(t, s, 0)$. For $s \geq 5$ the existence of a good Eulerian subgraph $W(t, s, 0)$ follows from the results in Case 3. The existence of a good Eulerian subgraph $W(t, 3,0)$ follows from Case 1.

Case 5: $s$ odd, $t$ odd, $t \geq 5$. A good Eulerian subgraph $W(s, t, 0)$ can be obtained from the graph $W(3,3,0)$ in Figure 6(a). If $r \in\{1,2\}$, then the existence of a good Eulerian subgraph follows from Proposition 6.4. Consider $3 \leq r \leq s-3$ and $s \geq 7$. Since $X(s, t, r)$ is isomorphic to $X(s, t, s-r)$ and $s$ is odd, we can construct 
a good Eulerian subgraph $W(s, t, r)$ for every $s, r$ odd, $s \geq 7,3 \leq r \leq s-4$. The graph $W(7,5,3)$ in Figure 10(c) can be expanded horizontally from column 4 and vertically from row 1 (or 2 ). It yields a good Eulerian subgraph $W(s, t, 3)$ for every $s, t$ odd, $s \geq 7, t \geq 5$. Since $s-r+3 \geq 7$, we can consider the graph $W(s-r+3, t, 3)$ arising from $W(7,5,3)$ in Figure 10(c). We expand horizontally the graph $W(s-r+3, t, 3)$ from column 2 and obtain a good Eulerian subgraph $W(s, t, r)$ for every $s, t, r$ odd, $s \geq 7, t \geq 5$ and $3 \leq r \leq s-4$.

Proposition 6.6. The graph $X(6 m+5,1,2), m \geq 0$, has no good Eulerian subgraph. Consequently, the generalized Petersen graph $G(6 m+5,2)$ has no Hamiltonian cycle.

Proof. We give a sketch of the proof by showing that $X(5,1,2)$ has no good Eulerian subgraph. Suppose, on the contrary, that $W$ is a good Eulerian subgraph of $X(6 m+5,1,2)$. Since the unique horizontal layer of $W$ has an odd number of vertices, the graph $W$ contains at least one path $P_{2 j+1}$ consisting of $2 j$ horizontal edges. It is possible to prove that $2 j=2$ (if $2 j>2$, then $W$ is not good). Without loss of generality we can set $P_{2 j+1}=$ $\left(x_{0}^{0}, x_{1}^{0}, x_{2}^{0}\right)$. Whence $\left[x_{3}^{0}, x_{4}^{0}\right] \in E(W)$ and no other horizontal edge of $X(5,1,2)$ belongs to $E(W)$. Moreover, $\left[x_{1}^{0}, x_{3}^{0}\right],\left[x_{1}^{0}, x_{4}^{0}\right]$ are edges of $W$, since $W$ is admissible and $x_{1}^{0}$ is 4 -valent in $W$. Whence $\left[x_{0}^{0}, x_{2}^{0}\right] \in E(W)$ and each admissible tour of $W$ contains the component $A=\left(x_{3}^{0}, x_{4}^{0}, x_{4}^{0}, x_{3}^{0}\right)$. That yields a contradiction, since $A$ is not a spanning subgraph of $X(6 m+5,1,2)$. Hence $X(5,1,2)$ has no good Eulerian subgraph. By Theorem 2.2, the graph $G(5,2)$ has no Hamiltonian cycle. The proof can be generalized to the case $G(6 m+5,2)$ with $m>0$.

\section{Characterization of Hamiltonian $I$-graphs}

Now we are ready to prove the main theorem.

Proof of Theorem 1.2. By Theorem 1.1, a generalized Petersen graph is Hamiltonian if and only if it is not isomorphic to $G(6 m+5,2), m \geq 0$. We prove that a proper $I$-graph is Hamiltonian. By Lemma 3.5, a proper $I$-graph $I(n, p, q)$ is special and its associated quartic graph $X$ is the circulant graph $\operatorname{Cir}(n ; p, q)$. By Lemma 5.1, the graph $\operatorname{Cir}(n ; p, q)$ can be represented as the graph $X(s, t, r)$, where $t=\operatorname{gcd}(n, q), s=n / t \geq 3, r \equiv$ $\pm p(q / t)^{-1}(\bmod s)$ and $(t, r) \neq(2,0)$ for odd values of $s$. By Lemma 6.5, the graph $X(s, t, r)$ has a good Eulerian subgraph. The assertion follows from Theorem 2.2.

By Theorem 2.2 and Lemma 6.5, we can extend the result of Theorem 1.2, about the existence of a Hamiltonian cycle, to the special generalized $I$-graphs.

As a consequence of Theorem 1.2, a proper $I$-graph is 3 -edge-colorable or, equivalently, 1-factorizable (because it is cubic and Hamiltonian). A widely studied property for 1-factorizable graphs is the property of admitting a perfect 1-factorization. We recall that a 1-factorization is perfect if the union of any pair of distinct 1-factors is a Hamiltonian cycle. Partial results are known for generalized Petersen graphs: $G(n, k)$ admits a perfect 1-factorization when $(n, k)=(3,1) ;(n, k)=(n, 2)$ with $n \equiv 3,4(\bmod 6)$; $(n, k)=(9,3) ;(n, k)=(3 d, d)$ with $d$ odd; $(n, k)=(3 d, k)$ with $k>1, d$ odd, $3 d$ and $k$ coprime (see [4]). So, it is quite natural to extend the same problem to proper $I$-graphs. 
Some further problems can be considered: the generalization of the existence of good Eulerian tour to other graph bundles of a cycle over a cycle, the characterization of Hamiltonian $G I$-graphs or of Hamilton-laceable $I$-graphs. In [8], the authors proved by a computer search that all bipartite connected $I$-graphs on $2 n \leq 200$ vertices are Hamilton-laceable.

\section{Appendix. Proof of Lemma 6.5}

Case $1, t=3$. We expand horizontally the graph $W(3,3,0)$ in Figure 6(a) from column 0 and obtain a good Eulerian subgraph $W(s, 3,0)$ for every $s$ odd, $s \geq 3$. A good Eulerian subgraph $W(s, 3,0)$ with $s$ even can be obtained from the graphs $W(4,3,0)$ and $W(6,3,0)$ in Figure 6(b)-(c). As an example, the graph $W(8,3,0)$ in Figure $7($ a) has been obtained by connecting two copies of the graph $W(4,3,0)$. The graph $W(10,3,0)$ in Figure $7(\mathrm{~b})$ has been obtained by connecting the graphs $W(4,3,0)$ and $W(6,3,0)$. For $r=1$ the existence of a good Eulerian subgraph $W(s, 3,1)$ follows from Proposition 6.4. Hence we can consider $2 \leq r \leq s / 2$, since $X(s, 3, r)$ is isomorphic to $X(s, 3, s-r)$. The graph $W(4,3,2)$ in Figure 7 (c) can be expanded horizontally from column 3 . It yields a good Eulerian subgraph $W(s, 3,2)$ for every $s$ even, $s \geq 4$. Since $s-r+2 \geq 4$, we can consider the graph $W(s-r+2,3,2)$ obtained from $W(4,3,2)$ in Figure 7 (c). We expand horizontally $W(s-r+2,3, r)$ from column 1 and obtain a good Eulerian subgraph $W(s, 3, r)$ for every $s, r$ even, $s \geq 4,2 \leq r \leq s / 2$. Analogously, the graphs $W(6,3,3)$, $W(8,3,3)$ and $W(10,3,5)$ in Figure 8 yield a good Eulerian subgraph $W(s, 3, r)$ for every $s$ even, $r$ odd, $3 \leq r \leq s / 2$. More specifically, we expand horizontally the graph $W(8,3,3)$ from column 7 and obtain a good Eulerian subgraph $W(s, 3,3)$ for every even integer $s \geq 8$. The graph $W(10,3,5)$ can be expanded horizontally from column 9 (or 0 ). It yields a good Eulerian subgraph $W(s, 3,5)$ for every even integer $s, s \geq 10$. Since $s-r+5 \geq 10$, we can consider the graph $W(s-r+5,3,5)$ obtained from $W(10,3,5)$ in Figure $8(\mathrm{c})$. We expand $W(s-r+5,3,5)$ from column 4 and obtain a good Eulerian subgraph $W(s, 3, r)$ for every $s$ even, $s \geq 10, r$ odd, $5 \leq r \leq s / 2$.

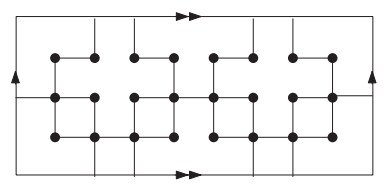

(a)

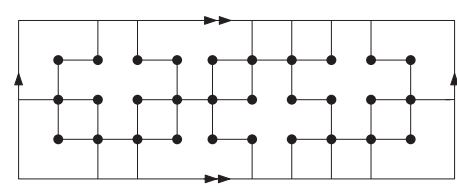

(b)

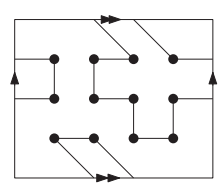

(c)

Figure 7: A good Eulerian subgraph: (a) $W(8,3,0)$; (b) $W(10,3,0)$; (c) $W(4,3,2)$.

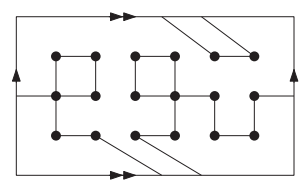

(a)

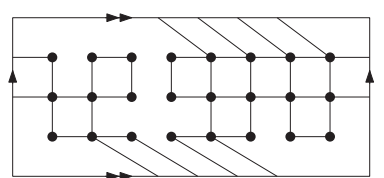

(b)

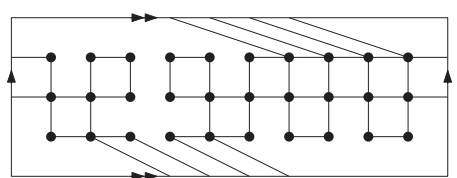

(c)

Figure 8: A good Eulerian subgraph: (a) $W(6,3,3)$; (b) $W(8,3,3)$; (c) $W(10,3,5)$.

Consider $s$ odd, $s \geq 5$. The graph $W(5,3,2)$ in Figure 9(a) can be expanded horizontally from column 4 . It yields a good Eulerian subgraph $W(s, 3,2)$ for every $s$ odd, 
$s \geq 5$. Analogously, the graph $W(9,3,4)$ in Figure 9 (b) yields a good Eulerian subgraph $W(s, 3,4)$ for every $s$ odd, $s \geq 9$. The graph $W(13,3,6)$ in Figure 9(c) can be expanded horizontally from column 2 and column 10. It yields a good Eulerian subgraph $W(2 r+1,3, r)$ with $r$ even, $6 \leq r \leq s / 2$. Since $s-2 r+1 \geq 0$, we can expand $W(2 r+1,3, r)$ from column $2 r$ and find a good Eulerian subgraph $W(s, 3, r)$ for every $s$ odd, $s \geq 13, r$ even, $r \geq 6$. It remains to construct a good Eulerian subgraph $W(s, 3, r)$ with $s, r$ odd, $s \geq 7,3 \leq r \leq s / 2$. We use the graph $W(7,3,3)$ in Figure 10 (a) to construct a good Eulerian subgraph $W(2 r+1,3, r)$ with $r$ odd, $r \geq 3$. As an example, the graph $W(11,3,5)$ in Figure 10(b) has been obtained by expanding horizontally the graph $W(7,3,3)$ from column $r=3$ and $s-1=6$ and by adding new diagonal edges. If we iterate the process, then we obtain a good Eulerian subgraph $W(2 r+1,3, r)$ with $r$ odd, $r \geq 3$. The graph $W(2 r+1,3, r)$ thus obtained can be expanded horizontally from column $2 r$. It yields a good Eulerian subgraph $W(s, 3, r)$ for every $s, r$ odd, $s \geq 7,3 \leq r \leq s / 2$.

(a)
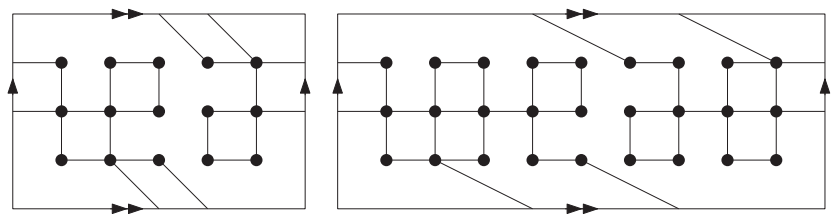

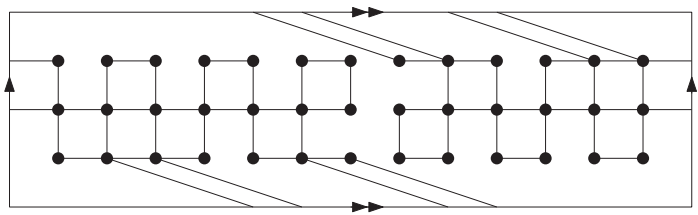

(c)

Figure 9: A good Eulerian subgraph: (a) $W(5,3,2)$; (b) $W(9,3,4)$; (c) $W(13,3,6)$

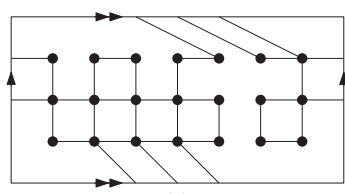

(a)

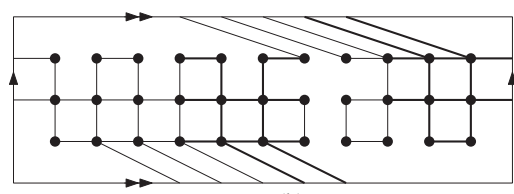

(b)

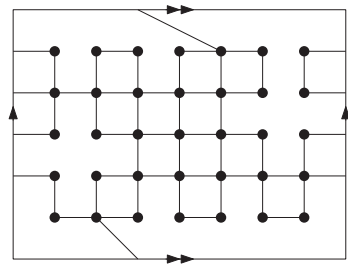

(c)

Figure 10: A good Eulerian subgraph: (a) $W(7,3,3)$; (b) $W(11,3,5)$; (c) $W(7,5,3)$. To obtain the graph $W(11,3,5)$ we expanded horizontally the graph $W(7,3,3)$ from column $r=3$ and column $s-1=6$, then we added new diagonal edges (see the bold edges).

\section{Acknowledgements}

The authors would like to thank Arjana Žitnik for careful reading of various versions of this paper and for many useful suggestions. 


\section{References}

[1] A. Ádám, Research problems, J. of Combin. Theory 2 (1967), 393 -, doi: http://dx.doi.org/10.1016/S0021-9800(67)80037-1, http: / / www. sciencedirect. $\mathrm{com} / \mathrm{science/article/pii/s0021980067800371.}$

[2] B. Alspach, The classification of Hamiltonian generalized Petersen graphs, J. Combin. Theory Ser. B 34 (1983), 293-312, doi:10.1016/0095-8956(83)90042-4, http: / / dx. doi.org/ $10.1016 / 0095-8956(83) 90042-4$.

[3] M. Boben, T. Pisanski and A. Žitnik, $I$-graphs and the corresponding configurations, J. Combin. Des. 13 (2005), 406-424, doi:10.1002/jcd.20054, http://dx.doi.org/10.1002/ jcd. 20054 .

[4] S. Bonvicini and G. Mazzuoccolo, Perfect one-factorizations in generalized Petersen graphs, Ars Combin. 99 (2011), 33-43.

[5] I. Z. Bouwer, W. W. Chernoff, B. Monson and Z. Star, The foster census, Charles Babbage Research Centre, Winnipeg (1988).

[6] M. D. E. Conder, T. Pisanski and A. Žitnik, $G I$-graphs: a new class of graphs with many symmetries, J. Algebraic Combin. 40 (2014), 209-231, doi:10.1007/s10801-013-0484-3, http: //dx.doi.org/10.1007/s10801-013-0484-3.

[7] C. Delorme, O. Favaron and M. Mahéo, Isomorphisms of Cayley multigraphs of degree 4 on finite abelian groups, European J. Combin. 13 (1992), 59-61, doi:10.1016/0195-6698(92) 90067-A, http://dx.doi.org/10.1016/0195-6698(92)90067-A.

[8] M. Dupuis and S. Wagon, Laceable knights, Ars Math. Contemp. 9 (2015), 115-124.

[9] B. Elspas and J. Turner, Graphs with circulant adjacency matrices, J. Combinatorial Theory 9 (1970), 297-307.

[10] C. Fan, D. R. Lick and J. Liu, Pseudo-Cartesian product and Hamiltonian decompositions of Cayley graphs on abelian groups, Discrete Math. 158 (1996), 49-62, doi: 10.1016/0012-365X(95)00035-U, http://dx.doi.org/10.1016/0012-365X (95) $00035-U$.

[11] H. Fleischner, (Some of) the many uses of Eulerian graphs in graph theory (plus some applications), Discrete Math. 230 (2001), 23-43, doi:10.1016/S0012-365X(00)00067-4, Paul Catlin memorial collection (Kalamazoo, MI, 1996), http://dx.doi.org/10.1016/ S0012-365X(00)00067-4.

[12] M. R. Garey, D. S. Johnson and R. E. Tarjan, The planar Hamiltonian circuit problem is NPcomplete, SIAM J. Comput. 5 (1976), 704-714.

[13] B. Horvat, T. Pisanski and A. Žitnik, Isomorphism checking of I-graphs, Graphs Combin. 28 (2012), 823-830, doi:10.1007/s00373-011-1086-2, http://dx.doi.org/10.1007/ s $00373-011-1086-2$.

[14] C. H. Li, On isomorphisms of finite Cayley graphs-a survey, Discrete Math. 256 (2002), 301-334, doi:10.1016/S0012-365X(01)00438-1, http://dx.doi.org/10 . 1016/S0012-365X(01)00438-1.

[15] D. Marušič and T. Pisanski, The remarkable generalized Petersen graph $G(8,3)$, Math. Slovaca 50 (2000), 117-121.

[16] M. Muzychuk, Ádám's conjecture is true in the square-free case, J. Combin. Theory Ser. A 72 (1995), 118-134, doi:10.1016/0097-3165(95)90031-4, http://dx.doi.org/10 . $1016 /$ $0097-3165$ ( 95 ) $90031-4$. 
[17] T. Pisanski and B. Servatius, Configurations from a graphical viewpoint, Birkhäuser Advanced Texts: Basler Lehrbücher. [Birkhäuser Advanced Texts: Basel Textbooks], Birkhäuser/Springer, New York, 2013, doi:10.1007/978-0-8176-8364-1, http://dx. doi . org/10.1007/978-0-8176-8364-1.

[18] T. Pisanski, T. W. Tucker and A. Žitnik, Straight-ahead walks in Eulerian graphs, Discrete Math. 281 (2004), 237-246, doi:10.1016/j.disc.2003.09.011, http://dx.doi .org/10 . $1016 / j . d i s c .2003 .09 .011$.

[19] T. Pisanski and J. Vrabec, Graph bundles, 1982, Preprint Series Dept. Math. Univ. Ljubljana 20 (1982), 213-298.

[20] P. Potočnik, P. Spiga and G. Verret, Cubic vertex-transitive graphs on up to 1280 vertices, $J$. Symbolic Comput. 50 (2013), 465-477, doi:10.1016/j.jsc.2012.09.002, http://dx. doi . org/10.1016/j.jsc.2012.09.002. 